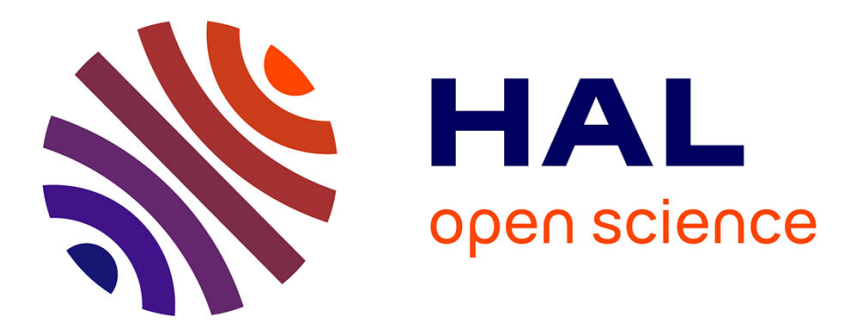

\title{
Microscopic and macroscopic models for vehicular and pedestrian flows
}

\author{
Massimiliano D. Rosini
}

\section{To cite this version:}

Massimiliano D. Rosini. Microscopic and macroscopic models for vehicular and pedestrian flows. Order, Disorder and Criticality: Advanced Problems of Phase Transition Theory. Ed. by Yu. Holovatch. Vol. 6, 2020, World Scientific, Singapore, 6, In press. hal-02491071

\section{HAL Id: hal-02491071 \\ https://hal.science/hal-02491071}

Submitted on 25 Feb 2020

HAL is a multi-disciplinary open access archive for the deposit and dissemination of scientific research documents, whether they are published or not. The documents may come from teaching and research institutions in France or abroad, or from public or private research centers.
L'archive ouverte pluridisciplinaire HAL, est destinée au dépôt et à la diffusion de documents scientifiques de niveau recherche, publiés ou non, émanant des établissements d'enseignement et de recherche français ou étrangers, des laboratoires publics ou privés. 


\title{
Chapter 1
}

\section{Microscopic and macroscopic models for vehicular and pedestrian flows}

\author{
Massimiliano D. Rosini \\ Dipartimento di Matematica e Informatica, Università degli Studi di Ferrara, \\ Via Machiavelli 35, 44121 Ferrara, Italy \\ and \\ Uniwersytet Marii Curie - Sktodowskiej, \\ Plac Marii Curie - Sktodowskiej 1, 20 - 031 Lublin, Poland, \\ massimilianodaniele.rosini@unife.it
}

\begin{abstract}
In this chapter we develop and study microscopic and macroscopic models for car and pedestrian flows. After a general introduction on the basic features of the flows under consideration, we develop our models. We first introduce at the microscopic level few basic and well established interaction rules. We then encode them in analytical models and obtain at the microscopic level a first order follow-the-leader model and at the macroscopic level a scalar conservation law (an hyperbolic partial differential equation). These models are then generalized to the cases of heterogeneous flows and flows across point-wise inhomogeneities characterized by reduced capacity, such as doors or toll gates. At last we also consider a crowd in panic situations. Some examples are proposed and serve as benchmarks to point out the ability of the models to reproduce realistic scenarios. The solutions to these examples are constructed by applying a wave-front tracking algorithm based on Riemann solvers. We also show how macroscopic models can be obtained from a microscopic model as many-particle limit.
\end{abstract}

\section{Contents}

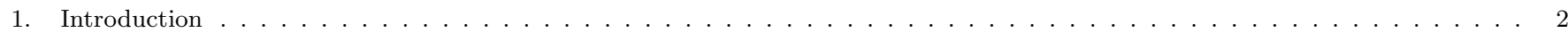

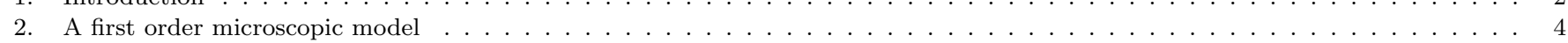

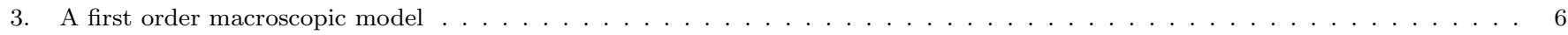

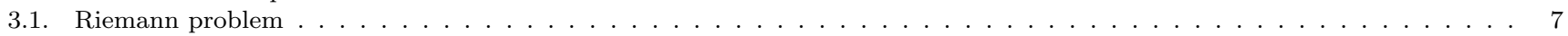

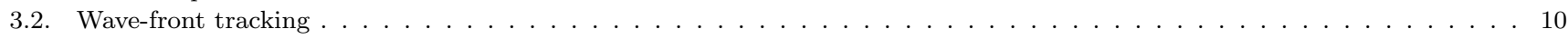

4. Links between microscopic and macroscopic models . . . . . . . . . . . . . . . . . . . . . . . . . . . . . . . 13

5. Heterogeneous traffic . . . . . . . . . . . . . . . . . . . . . . . . . . . . . . . . . . . 15

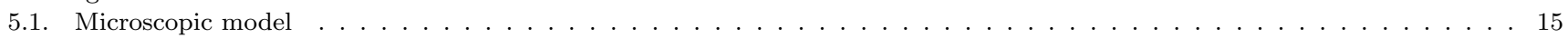

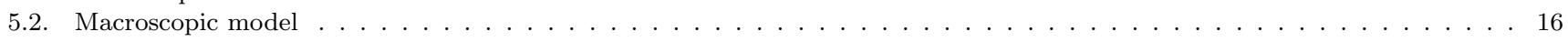

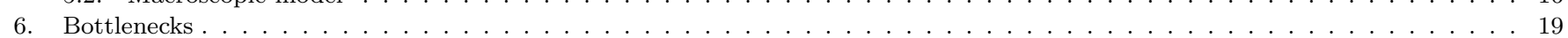

6.1. Microscopic model . . . . . . . . . . . . . . . . . . . . . . . . . . . . . . . . . . . . . 19

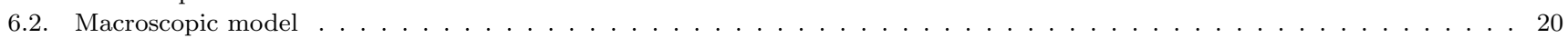

7. A macroscopic model for pedestrian flows with panic . . . . . . . . . . . . . . . . . . . . . . . . . . . . . . . . 24

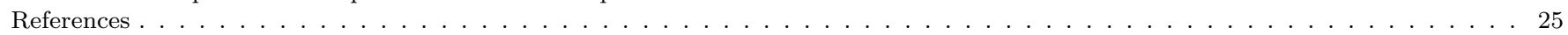


February 25, 2020 16:29 Preprint-9x6 Book Title: Order, Disorder and Criticality: Advanced Problems of Preprint page 2 Phase Transition Theory. Ed. by Yu. Holovatch. Vol. 6, 2020, World Scientific, Singapore

\section{Introduction}

In this chapter we consider both car and pedestrian flows. To some extent, they are well known from every day life experiences. For this reason, there is no need to give a technical description, as some general insights are already well-known to the reader. Nevertheless, there are some counter intuitive features that appear as paradoxes and need some comments.

This chapter aims at indicating research perspectives on car and pedestrian flows motivated by insights into their complexity features. In fact drivers and crowds have to be looked at as living complex systems, namely systems of agents who are able to choose actively their individual dynamics. As a consequence, the emergent collective behavior and self-organization are not generated by a passive reaction of the whole system to external stimuli, but they stem from one-to-one, or at most one-to-few, interactions among the agents.

Consider the evacuation of a room through an exit door as in Fig. 1. The presence of an exit door has a

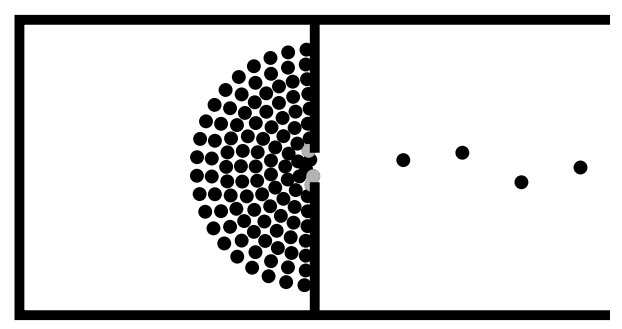

Fig. 1.: Capacity drop of an exit door. The dots represent a crowd evacuating a $2 \mathrm{D}$ room with a single exit door. The dots marked in gray are clogging the exit door.

big impact on the evacuation time. An important property of the door is its capacity $Q$, which gives the maximum number of pedestrians that can flow through it in a given time interval. Despite the common belief, the capacity of a door is not constant. In fact high densities upstream of an exit door may determine its capacity drop. This is clear by observing that two or more pedestrians may block each other and not move further. In real situations these kind of clogging are avoided by placing a further door or column in the upstream vicinity of the door. Indeed, the presence of such obstacles may prevent the crowd density from reaching high values and may help to minimize the evacuation time, since in a moderate density regime the full capacity of the exit door can be exploited. The decrease of the evacuation time may seem unexpected, as some of the pedestrians are forced to choose a longer path to reach the exit. This is usually referred to as Braess' paradox for pedestrians.

A further phenomenon linked to the capacity drop of the exit doors is the so-called faster-is-slower effect. ${ }^{2}$ Faster-is-slower effect refers to the jamming and clogging at the doors, that result in an increase of the evacuation time when the velocity of a crowd is high. ${ }^{3,4}$ This partly counter-intuitive phenomenon is explained by the fact that if a huge number of pedestrians reach the exit door faster, then the capacity of the exit door falls earlier.

In fact, the importance of these paradoxes stems from the fact that they serve as benchmarks to point out the ability of the models to reproduce realistic scenarios. We aim to develop microscopic and macroscopic models able to reproduce both the Braess' paradox and the fasteris-slower effect. In the literature, there are two main approaches to do so. The first just couples the equations for the evolution of the crowd, with boundary conditions corresponding to obstacles, such as walls and doors, see for instance Ref. [5-7]. The second approach adds a further condition which accounts for the capacity of the bottlenecks, as in the present chapter and in Ref. [8-11].

In Section 6 we reproduce the effects of an exit doors by applying the theory of point constraint on the flow first introduced in Ref. [8]. More precisely, in Examples 22 and 23 we reproduce the Braess' paradox and the fasteris-slower effect.

Since traffic dynamics are essentially microscopic, it is much easier to physically motivate the microscopic rather than some macroscopic assumptions. Thus, we first encode the interacting rules in a first order microscopic follow-the-leader (FTL) model. We then construct the first order macroscopic Lighthill-Whitham-Richards (LWR) model ${ }^{12,13}$ taking advantage of analogies with the first order FTL model. We also recall that in Ref. [14] it is rigorously proved that LWR model can be obtained as many-particle limit of FTL model approximations, similar to taking the hydrodynamical limit of Boltzmann equations

We then propose some possible generalizations. In the case of a heterogeneous traffic with vehicles charac- 
terized in terms of length and maximal speed, we show how to deduce at the microscopic level a FTL model and at the macroscopic level the Aw-Rascle-Zhang (ARZ) model. ${ }^{15,16}$ We point out the analogies between the two models and recall that in Ref. [17] it is rigorously proved that ARZ model can be obtained as many-particle limit of FTL model approximations. We then consider the presence of pointwise inhomogenities. From the modeling point of view, pointwise inhomogenities correspond, for instance, to a narrow exit in crowd modeling, or to a toll gate in vehicular traffic. This leads us to consider point constraints on the flow. At the macroscopic level this is encoded by simply adapting the velocity function. At the macroscopic level, we rely on the paradigm of passage to the limit from microscopic to macroscopic level proposed in Ref. [18]. In particular we give two examples that show the ability of the model to reproduce two paradoxes linked to the presence of bottlenecks, namely Braess' paradox and faster-is-slower effect. At last we also develop a macroscopic model for crowd dynamics in case of panic, ${ }^{8}$ which is able to reproduce the arise of panic.

Vehicular and pedestrian flows share most of their main features. The main difference is the panic behavior that characterizes only crowd dynamics. Panic is one of the main cause of crowd accidents. We make clear some basic features of such events. First, during a crowd accident compressive forces occur from both vertical stacking and horizontal pushing, moreover the domino effect of people leaning against each other has to be taken into account. Evidence of bent steel railings after fatal crowd accidents show that forces of more than $4,500 \mathrm{~N}$ occurred. For instance, at Ibrox Park soccer stadium in UK, after the crowd accident occurred in 1971, the pile of bodies was $3 m$ high, showing that people were moving over each other. Second, deaths from human stampedes occur primarily from compressive asphyxiation and not trampling. For instance after the crowd accident occurred at Riverfront Coliseum in Cincinnati, USA, on 1979, a line of bodies was found $9 \mathrm{~m}$ from a wall. This indicates that crowd pressures came from both directions as rear ranks pressed forward and front ranks pushed off the wall.

Crowd accidents are caused by an irrational behavior of pedestrians due to panic. According to sociology scientists, panic is a maladaptive reaction of flight stemming from intense fear. Moreover, panic is infectious and easily spreads to other people nearby. Such definition cannot be easily exploited in an analytical model. For this reason in this chapter panic simply corresponds to densities higher than (7 people) $/ \mathrm{m}^{2}$. This choice stems from the fact that we want to avoid deaths that result from panic, namely from high densities.

Crowd behaviour is not always the primary cause of accidents. Architects and city planners try to provide a safe environment for places of public assembly by using analytical models to determine and prevent congestion. A good example is the Jamarat Bridge, Saudi Arabia. Following the 2006 accident with 363 deaths, see also Table 1 , the old bridge was demolished and construction began on a new 4-level bridge. The new bridge is designed to accommodate 5 million pilgrims. It has 10 entrances and 12 exits to allow the flow of 300,000 pilgrims per hour. It is also equipped with surveillance technology to help authorities intervene in case of any stampedes. As a result, so far no crowd accidents occur on the Jamarat Bridge since $2006 .^{\mathrm{a}}$

Table 1.: Some notable crowd accidents occurred at the Jamarat Bridge, Saudi Arabia.

\begin{tabular}{cccccccc}
\hline Year & 2006 & 2004 & 2001 & 1998 & 1997 & 1994 & 1990 \\
\hline Dead & 363 & 249 & 35 & 118 & 22 & 266 & 1,426 \\
\hline
\end{tabular}

It is therefore possible to avoid or to mitigate crowd accidents. Nowadays, all public entertainment venues are equipped with doors that open outwards using crash bar latches that open when pushed. Moreover, it is well known since ancient times, that a tall column placed in the upstream of the door exit may reduce the interpedestrian pressure, decreasing the magnitude of clogging and making the overall outflow higher and more regular, this is the so called Braess' paradox that we have already mentioned.

Vehicular and pedestrian flows can be described at three levels: microscopic, mesoscopic and macroscopic. At the microscopic level we work in the framework of Newtonian mechanics and the dynamics of each pedes-

an 2015 around 1,470 deaths occur in the camps close to the Jamarat Bridge, so it cannot be directly linked to a failure of the new bridge. 
trian moving under the action of the surrounding pedestrians and of the environment is represented by an ordinary differential equation. Examples of microscopic models are follow-the-leader models, ${ }^{19}$ AI-based models, ${ }^{20}$ social force model, ${ }^{21}$ cellular automata models. ${ }^{22,23}$ At the mesoscopic level we work in the framework of kinetic theory of gases and introduce a Boltzmann-type evolution equation for the statistical distribution function of the position and velocity of the pedestrians, see for example Ref. [24-26] and references therein. At the macroscopic level we work in the framework of the hydrodynamics and the flow is described in terms of averaged quantities, which are assumed to be continuous and evolve according to partial differential equations. The first macroscopic modeling attempt is due to Hughes. ${ }^{27}$ Current macroscopic models use non linear conservation laws with non classical shocks, ${ }^{8}$ gas dynamics equations, ${ }^{28,29}$ time evolving measures ${ }^{30}$ and gradient flow methods. ${ }^{31}$

We concentrate our attention on microscopic and macroscopic models. On one hand there are a posteriori some advantages in using macroscopic models. For instance, it is very powerful in the description of queue tails as they correspond to the position of shock waves. Furthermore it is suitable with very large number of cars or pedestrians as it is less computationally intensive than microscopic models and can allow fast simulations also for large-scale traffic networks. Moreover it is easy to validate and implement due to the low number of parameters. At last is it suitable to real time prediction, estimation, optimization and management. On the other hand, the number of cars or pedestrians is far lower than that of molecules, for instance, in gas dynamics. Therefore the continuum assumption is not justified and the macroscopic formulation is not a priori justified. In this respect, a validation of the macroscopic approach is provided by the micro-macro limit rigorously proved in Ref. [14].

At the macroscopic level the flow of cars or pedestrians is seen as a unique stream, in analogy with the flow of fluids or gases, and its dynamics are described by means of aggregate variables, such as density, mean speed and flow. Hence macroscopic models describe the traffic as a fluid of "thinking" particles. However we have to keep in mind the following differences between the flow of cars or pedestrians and the flow of fluids:

(1) As we already noticed, the continuum assumption is not justified in the framework of car or pedestrian flows, because the number of vehicles or pedestrians is far lower than that of molecules, hence vehicles or pedestrians do not form a continuum.

(2) A fluid particle is isotropic (i.e., responds to stimuli from the surrounding particles), whereas a vehicle or a pedestrian is anisotrpic (i.e., responds only to frontal stimuli).

(3) Vehicles and pedestrians don't have negative speed.

(4) In vehicular and pedestrian flows there is no conservation of momentum.

(5) A driver or a pedestrian is a living system since he/she has a personality (e.g., aggressive or timid) and this remains unchanged by motion (e.g., a slow driver is unaffected by its interaction with faster drivers passing it, or queueing behind it).

This chapter is structured as follows. In the next section we construct a first order microscopic model starting from basic considerations. In Section 3 we construct a first order macroscopic model, that is the LWR model, and show how the wave-front tracking algorithm can construct a weak solution on the basis of a Riemann solver. We give two examples and show that the obtained weak solutions are realistic. We conclude the section by pointing out the links between the two first order models. In Section 5 we generalize the previous two models to the case of an heterogeneous traffic and obtain a second order FTL model and the ARZ model. As in the previous section, we give an example and show that the obtained weak solution is realistic. In Section 6 we take into consideration the effects of pointwise bottlenecks, both at microscopic and macroscopic levels. We reproduce the Braess' paradox and the faster-is-slower effect with two examples. We also point out further possible generalizations. At last in Section 7 we propose a way to construct a macroscopic model for the evacuation of a narrow corridor in the case of panic behavior.

\section{A first order microscopic model}

Aim of this section is to develop our first microscopic model for vehicular and pedestrian flows starting from basic interaction rules. For simplicity in the exposition, we refer to car traffic; the case with pedestrians is analogous. 
Consider the simplest case of a straight one-lane homogeneous road, along which overtaking is not allowed. The road can be parametrized by $x \in \mathbb{R}$. If we have $n$ cars, then traffic is described by the position of each car at each time, namely by

$$
x_{1}(t), x_{2}(t), \ldots, x_{i}(t), \ldots, x_{n}(t) \in \mathbb{R} .
$$

More precisely $x_{i+1}(t)<x_{i}(t)$ and $x_{i}(t)$ corresponds to the rear of the car. It is not restrictive to assume that cars move with positive speed, see Fig. 2.

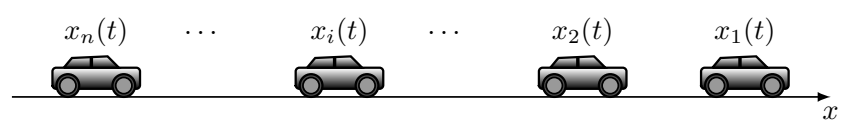

Fig. 2.: A car traffic.

The simplest case is when the traffic is given by just one car, i.e. $n=1$. In this case such car has free road, it moves at the maximum speed $v_{\max }>0$ and its equation of motion is

$$
\dot{x}_{1}(t)=v_{\max },
$$

where $\dot{x}_{1}$ is the time derivative of position $x_{1}$. Equation (1) is a first order linear ordinary differential equation (ODE). In order to know the (exact) position of the car at each time, it is necessary to know its position at an arbitrarily fixed time. If for instance we know that

$$
x_{1}\left(t_{0}\right)=\bar{x}_{1},
$$

where $\bar{x}_{1} \in \mathbb{R}$ is the position of the car at time $t_{0} \in \mathbb{R}$, then its position at time $t \in \mathbb{R}$ is given by

$$
x_{1}(t)=\bar{x}_{1}+\left(t-t_{0}\right) v_{\max } .
$$

We just solved the Cauchy problem (1), (2), indeed its (unique) solution is given by (3).

We consider now a second car which at time $t_{0}$ is at $\bar{x}_{2} \in \mathbb{R}$, namely

$$
x_{2}\left(t_{0}\right)=\bar{x}_{2} .
$$

It is not restrictive to assume that $\bar{x}_{2}<\bar{x}_{1}$ (otherwise we just change the labels of the cars). More precisely, if both cars have length $\ell$, then we assume that

$$
\bar{x}_{1}-\bar{x}_{2} \geqslant \ell \text {. }
$$

In principle we could think that also the second car moves at the maximum speed $v_{\max }$. This would not lead to any contradiction as cars will not overtake each other.
What's more, this would definitely optimize the traffic flow! However this is in contrast with reality, as (nonautonomous) cars move taking into account their distance from the car ahead, which is called in jargon its leader. For this reason it is more reasonable to consider an equation of motion of the form

$$
\dot{x}_{2}(t)=v\left(\frac{1}{x_{1}(t)-x_{2}(t)}\right),
$$

for a "well-chosen" function $v$. Equation (5) is a first order non-linear ODE.

The explicit expression for $v$ can be obtained from experimental data. Bruce D. Greenshields was the first to

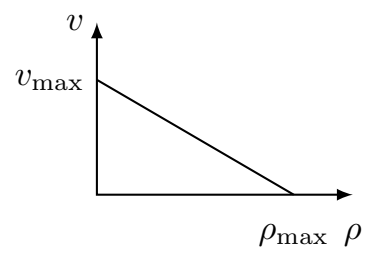

Fig. 3.: A linear velocity function.

carry out tests to measure traffic flow, traffic density and speed using photographic measurement methods in the 1930s. He postulated a linear relationship between speed and traffic density as in Fig. 3. Nowadays technology allows to have more advanced and detailed experimental data. As a result it is nowadays clear that the speeddensity diagram is in general given by a cloud of points, it depends on the lane of the road and also on the time interval of the day under consideration.

Since we aim to develop a reasonably general model that can be applied to different cases, we rather have to understand which conditions must be imposed on $v$ in order to obtain a realistic model. Let's start from the following basic considerations:

(1) overtaking is not allowed and the minimal distance between two cars is $\ell$, hence the argument of $v$ in (5) ranges in $\left[0, \rho_{\max }\right]$, where $\rho_{\max }=1 / \ell$;

(2) speeds cannot be negative and are bounded from above by $v_{\max }$, hence $v$ takes values in $\left[0, v_{\max }\right]$;

(3) higher velocities correspond to higher distances from the car ahead, hence $v$ is decreasing;

(4) a car is at rest if and only if it is "bumper-to-bumper" with the car ahead, see Fig. 4 , namely $x_{1}(t)-x_{2}(t)=$ $\ell$, hence $v(\rho)=0$ if and only if $\rho=\rho_{\max }$. 


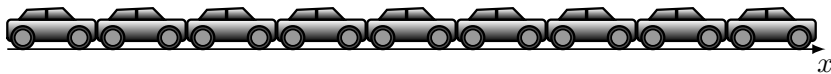

Fig. 4.: A "bumper-to-bumper" traffic condition. As a consequence, we are led to assume the following hypothesis

$$
\begin{aligned}
& v:\left[0, \rho_{\max }\right] \rightarrow\left[0, v_{\max }\right] \text { is a de- } \\
& \text { creasing function such that } v(0)= \\
& v_{\max } \text { and } v\left(\rho_{\max }\right)=0 .
\end{aligned}
$$

Clearly, a linearly decreasing velocity as in Fig. 3 satisfies $(\mathrm{V})$.

In summary, the model for a traffic given by two cars is described by Equations (1), (2), (4) and (5), namely by the Cauchy problem

$$
\left\{\begin{array}{l}
\dot{x}_{1}(t)=v_{\max }, \\
\dot{x}_{1}(t)=v\left(\frac{1}{x_{1}(t)-x_{2}(t)}\right), \\
x_{i}(0)=\bar{x}_{i},
\end{array} \quad i \in\{1,2\},\right.
$$

with $v$ satisfying (V). By iterating (4) and (5) it is now clear that the traffic given by $n \geqslant 2$ cars is described by the Cauchy problem for a first order system of non-linear ODEs

$$
\begin{cases}\dot{x}_{1}(t)=v_{\max } & \\ \dot{x}_{i}(t)=v\left(\frac{1}{x_{i-1}(t)-x_{i}(t)}\right), & i \in\{2, \ldots, n\}, \\ x_{i}(0)=\bar{x}_{i}, & i \in\{1, \ldots, n\} .\end{cases}
$$

Model (6) is called first order follow-the-leader (FTL) model. Its name recalls that the speed of each car depends only on its distance from the car ahead (its leader) and because it is a system of first order ODEs.

\section{A first order macroscopic model}

The macroscopic variables are the density $\rho$, that is the number of vehicles per unit length, the velocity $v$, that is the space covered per unit time, and the flow $f$, that is the number of vehicles per unit time. We show now how to formally derive the relations between $\rho, v$ and $f$.

Example 1. Consider cars with the same length $\ell$ and moving equally spaced with the same velocity $v$, see Fig. 5. Both the distance between vehicles and the density $\rho$ do not change in time. Thus the number of vehicles passing an observer at $x_{0} \in \mathbb{R}$ in the time interval $[0, \tau]$

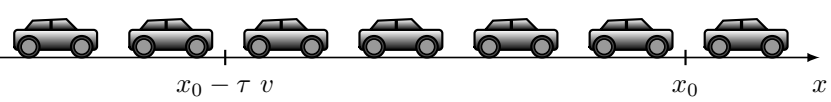

Fig. 5.: The case considered in Example 1. is the number of vehicles initially in the space interval $\left[x_{0}-\tau v, x_{0}\right]$ and therefore

$$
f=\frac{\rho\left(x_{0}-\left(x_{0}-\tau v\right)\right)}{\tau} \quad \Longrightarrow \quad f=\rho v .
$$

Example 2. Consider in the time interval $\left[t_{1}, t_{2}\right]$ a section of the road $[a, b]$ with neither entries nor exits. Clearly, the number of cars in $[a, b]$ at time $t=t_{2}$ is equal to the number of cars in $[a, b]$ at time $t=t_{1}$, plus the number of cars that are entering in the time interval $\left[t_{1}, t_{2}\right]$ through $x=a$, minus the number of cars that are exiting in the time interval $\left[t_{1}, t_{2}\right]$ through $x=b$, see Fig. 6. In formulas this means that

$$
\int_{a}^{b} \rho\left(t_{2}, y\right) \mathrm{d} y=\int_{a}^{b} \rho\left(t_{1}, y\right) \mathrm{d} y+\int_{t_{1}}^{t_{2}} f(t, a) \mathrm{d} t-\int_{t_{1}}^{t_{2}} f(t, b) \mathrm{d} t .
$$

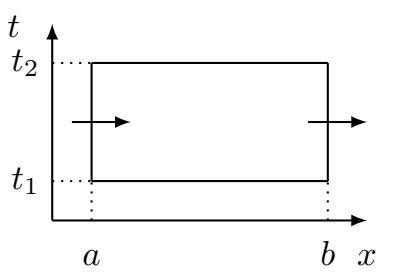

Fig. 6.: The case considered in Example 2.

Then, at least formally, we derive that

$$
\int_{t_{1}}^{t_{2}} \int_{a}^{b}\left(\partial_{t} \rho+\partial_{x} f(\rho)\right) \mathrm{d} x \mathrm{~d} t=0 .
$$

Since $a, b, t_{1}$ and $t_{2}$ are arbitrary, the integrand must be null, namely

$$
\partial_{t} \rho+\partial_{x} f=0
$$

We just obtained a scalar conservation law expressing the conservation of the number of cars. It is a partial differential equation (PDE).

We have then two equations (7), (8) for the three unknown variables $\rho, v$ and $f$. Therefore we need a further condition. However any other condition results from an approximation of empirical observations and has no a priori justification. 
The simplest way to close the system is by assuming that the velocity of each vehicle depends on the density alone

$$
v=v(\rho) .
$$

The considerations done in the previous section lead us to assume that the velocity function $v:\left[0, \rho_{\max }\right] \rightarrow\left[0, v_{\max }\right]$ satisfies $(\mathrm{V})$. It is standard by now to quantify macroscopic properties of traffic flow in terms of the fundamental diagram, which relates the traffic flow measured in cars per unite time and the traffic density measured in cars per unit length. The reason is that a fundamental diagram shows beside the flow $f(\rho)$, also the velocity as $v(\rho)$ is by definition the slope of the line joining the origin and the point $(\rho, f(\rho))$ on the fundamental diagram, see Fig. 7. Below we display such diagrams for different cases.

As a result, we obtain the scalar conservation law

$$
\partial_{t} \rho+\partial_{x}(\rho v(\rho))=0 .
$$

We just deduced the Lighthill-Whitham-Richards (LWR) model. ${ }^{12,13}$
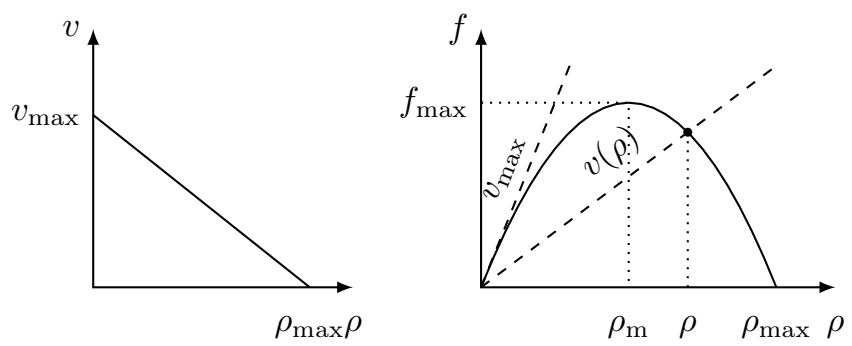

Fig. 7.: On the left a linear velocity function. On the right the corresponding strictly concave fundamental diagram. Notice that the slope of the tangent to the fundamental diagram is $v_{\max }$. Moreover $v(\rho)$ is the slope of the line crossing the fundamental diagram at $(0,0)$ and $(\rho, f(\rho))$.

In the remaining of this section we show how to approximate solutions to Cauchy problems for LWR model (9) by considering simple Riemann problems. This method is called wave-front tracking method. ${ }^{32-34}$ We recall that a Riemann problem is a Cauchy problem with piecewise constant initial datum with (at most) only one jump.
For simplicity we assume below that $v:\left[0, \rho_{\max }\right] \rightarrow$ $\left[0, v_{\max }\right]$ is $\mathbf{C}^{\mathbf{2}}$ and such that $f(\rho)=\rho v(\rho)$ is strictly concave, namely

$$
f^{\prime \prime}(\rho)=2 v^{\prime}(\rho)+\rho v^{\prime \prime}(\rho)<0 \quad \forall \rho \in\left[0, \rho_{\max }\right] .
$$

\subsection{Riemann problem}

Consider the Riemann problem for LWR model

$$
\left\{\begin{array}{l}
\partial_{t} \rho+\partial_{x} f(\rho)=0, \\
\rho(0, x)= \begin{cases}\rho_{L} & \text { if } x<0, \\
\rho_{R} & \text { if } x \geqslant 0,\end{cases}
\end{array}\right.
$$

where $f(\rho)=\rho v(\rho)$ and $\rho_{L}, \rho_{R} \in\left[0, \rho_{\max }\right]$ are constants. The invariance of both PDE and initial datum under the change of coordinates

$$
(t, x) \mapsto(\alpha t, \alpha x), \quad \alpha>0,
$$

suggests to look for solutions invariant under such change of coordinates, namely that are constant along the rays $x=m t, m \in \mathbb{R}$. Hence we restrict ourself to self-similar solutions, namely to solutions of the form

$$
\rho(t, x)=\phi(x / t) \text {. }
$$

This change of variables formally reduces the PDE in (10) to the following ODE

$$
0=-\frac{x}{t^{2}} \dot{\phi}+\frac{1}{t} f^{\prime}(\phi) \dot{\phi}=\frac{1}{t}\left(f^{\prime}(\phi)-\frac{x}{t}\right) \dot{\phi} .
$$

By defying $\xi=x / t$, Riemann problem (10) becomes then the limiting problem

$$
\left\{\begin{array}{l}
\left(f^{\prime}(\phi)-\xi\right) \dot{\phi}=0, \\
\phi(-\infty)=\rho_{L}, \\
\phi(+\infty)=\rho_{R}
\end{array}\right.
$$

We need to distinguish the following cases to construct a solution to (11):

- If $\rho_{L}=\rho_{R}$, then the constant function $\phi \equiv \rho_{L, R}$ is the solution.

- If $\rho_{L} \neq \rho_{R}$, then $\dot{\phi} \not \equiv 0$ and $f^{\prime}(\phi(\xi))=\xi$. By this last condition and the strict concavity of $f$ we have

$$
\begin{aligned}
f^{\prime}\left(\phi\left(f^{\prime}(\rho)\right)\right)=f^{\prime}(\rho) & \Longrightarrow \phi\left(f^{\prime}(\rho)\right)=\rho \\
& \Longrightarrow \phi(\xi)=\left(f^{\prime}\right)^{-1}(\xi) .
\end{aligned}
$$

By the conditions at the infinity and the strict con- 
February 25, 2020 16:29 Preprint-9×6 Book Title: Order, Disorder and Criticality: Advanced Problems of Preprint page 8 Phase Transition Theory. Ed. by Yu. Holovatch. Vol. 6, 2020, World Scientific, Singapore

cavity of $f$ we have then

$$
\begin{aligned}
\rho_{L} & =\phi(-\infty)=\left(f^{\prime}\right)^{-1}(-\infty)> \\
& >\left(f^{\prime}\right)^{-1}(+\infty)=\phi(+\infty)=\rho_{R} .
\end{aligned}
$$

In conclusion we get the continuous and piecwise $\mathbf{C}^{\mathbf{1}}$ solution

$$
\phi(\xi)= \begin{cases}\rho_{L} & \text { if } \xi<f^{\prime}\left(\rho_{L}\right), \\ \left(f^{\prime}\right)^{-1}(\xi) & \text { if } f^{\prime}\left(\rho_{L}\right) \leqslant \xi<f^{\prime}\left(\rho_{R}\right), \\ \rho_{R} & \text { if } \xi \geqslant f^{\prime}\left(\rho_{R}\right),\end{cases}
$$

at least in the case $\rho_{L}>\rho_{R}$.

- If $\rho_{L}<\rho_{R}$, then we have to look for discontinuous solutions and consider the weak formulation of $\left(f^{\prime}(\phi)-\xi\right) \dot{\phi}=0$, that is

$$
\int_{\mathbb{R}}\left(f(\phi) \varphi^{\prime}-\phi \frac{\mathrm{d}}{\mathrm{d} \xi}(\xi \varphi)\right) \mathrm{d} \xi=0 \quad \forall \varphi \in \mathbf{C}_{\mathbf{c}}^{\infty}\left(\mathbb{R}^{2} ; \mathbb{R}\right) .
$$

The expression of the initial datum suggests to look for a weak solution with a single jump. By introducing

$$
\phi(\xi)= \begin{cases}\rho_{L} & \text { if } \xi<\sigma \\ \rho_{R} & \text { if } \xi \geqslant \sigma\end{cases}
$$

in (12) we obtain the constant $\sigma \in \mathbb{R}$ as follows:

$$
\begin{gathered}
\int_{-\infty}^{\sigma}\left(f\left(\rho_{L}\right) \varphi^{\prime}-\rho_{L} \frac{\mathrm{d}}{\mathrm{d} \xi}(\xi \varphi)\right) \mathrm{d} \xi \\
+\int_{\sigma}^{\infty}\left(f\left(\rho_{R}\right) \varphi^{\prime}-\rho_{R} \frac{\mathrm{d}}{\mathrm{d} \xi}(\xi \varphi)\right) \mathrm{d} \xi=0 \\
\Leftrightarrow\left(\left(f\left(\rho_{L}\right)-f\left(\rho_{R}\right)\right)-\left(\rho_{L}-\rho_{R}\right) \sigma\right) \varphi(\sigma)=0 \\
\Leftrightarrow \sigma=\frac{f\left(\rho_{L}\right)-f\left(\rho_{R}\right)}{\rho_{L}-\rho_{R}} .
\end{gathered}
$$

Therefore (13) is a piecewise constant weak solution with $\sigma$ given above in the case with $\rho_{L}<\rho_{R}$.

Remark 3. The above computations for the speed of propagation of a discontinuity hold also in the case $\rho_{L}>$ $\rho_{R}$. For this reason, any discontinuity $\left(\rho_{L}, \rho_{R}\right)$ with $\rho_{L} \neq$ $\rho_{R}$ have speed of propagation

$$
\sigma\left(\rho_{L}, \rho_{R}\right)=\frac{f\left(\rho_{L}\right)-f\left(\rho_{R}\right)}{\rho_{L}-\rho_{R}} .
$$

Such condition is the so called Rankine-Hugoniot condition. ${ }^{35}$

We just obtained the Riemann solver $\mathcal{R}_{\mathrm{LWR}}$, which is an operator that associates to any $\rho_{L}, \rho_{R} \in\left[0, \rho_{\max }\right]$ the weak solution $\phi(\xi)=\mathcal{R}_{\mathrm{LWR}}\left[\rho_{L}, \rho_{R}\right](\xi)$ of the corresponding Riemann problem (11) constructed above; we define it more rigorously in the following definition. Below we denote by BV the space of functions with bounded variation. We can assume that any function in $\mathbf{B V}$ is right continuous by possibly changing its values at countably many points.

Definition 4. The Riemann solver $\mathcal{R}_{\mathrm{LWR}}:\left[0, \rho_{\max }\right]^{2} \rightarrow$ $\mathbf{B V}\left(\mathbb{R} ;\left[0, \rho_{\text {max }}\right]\right)$ associated to LWR model $(9)$ is defined as follows:

(1) If $\rho_{L}=\rho_{R}$ then $\mathcal{R}_{\mathrm{LWR}}\left[\rho_{L}, \rho_{R}\right] \equiv \rho_{L, R}$.

(2) If $\rho_{L}>\rho_{R}$ then $\mathcal{R}_{\mathrm{LWR}}\left[\rho_{L}, \rho_{R}\right]$ is the rarefaction wave

$\mathcal{R}_{\mathrm{LWR}}\left[\rho_{L}, \rho_{R}\right](\xi)= \begin{cases}\rho_{L} & \text { if } \xi<f^{\prime}\left(\rho_{L}\right) t, \\ \left(f^{\prime}\right)^{-1}(\xi) & \text { if } f^{\prime}\left(\rho_{L}\right) \leqslant \xi<f^{\prime}\left(\rho_{R}\right), \\ \rho_{R} & \text { if } \xi \geqslant f^{\prime}\left(\rho_{R}\right) .\end{cases}$

(3) If $\rho_{L}<\rho_{R}$ then $\mathcal{R}_{\mathrm{LWR}}\left[\rho_{L}, \rho_{R}\right]$ is the shock wave

$$
\mathcal{R}_{\mathrm{LWR}}\left[\rho_{L}, \rho_{R}\right](\xi)= \begin{cases}\rho_{L} & \text { if } \xi<\sigma\left(\rho_{L}, \rho_{R}\right), \\ \rho_{R} & \text { if } \xi \geqslant \sigma\left(\rho_{L}, \rho_{R}\right),\end{cases}
$$

where $\sigma\left(\rho_{L}, \rho_{R}\right)$ is the speed of propagation of the discontinuity and is given by (14).
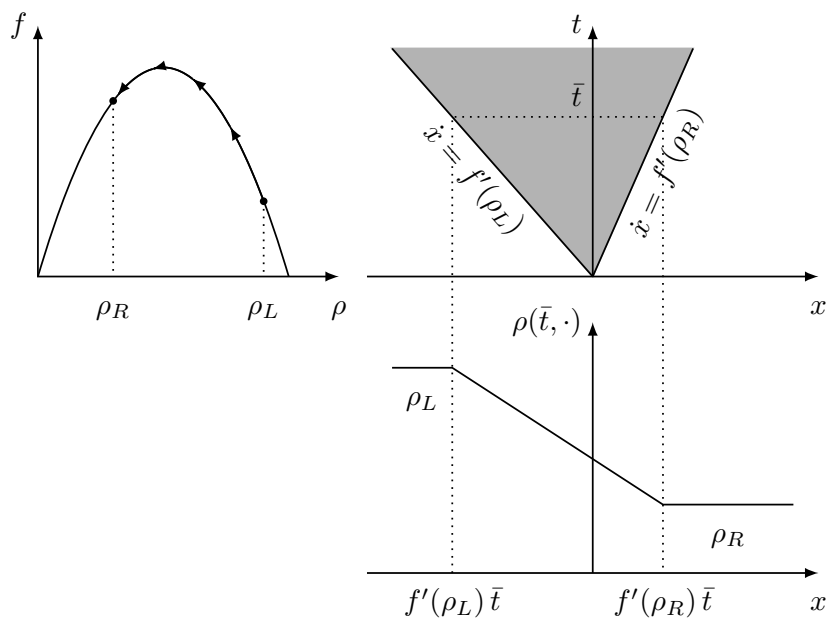

Fig. 8.: A rarefaction wave. On the left we represent the fundamental diagram. On the right we give the solution in the $(x, t)$-plane, above, and its profile $x \mapsto \rho(\bar{t}, x)$ computed at a time $\bar{t}>0$, below. 

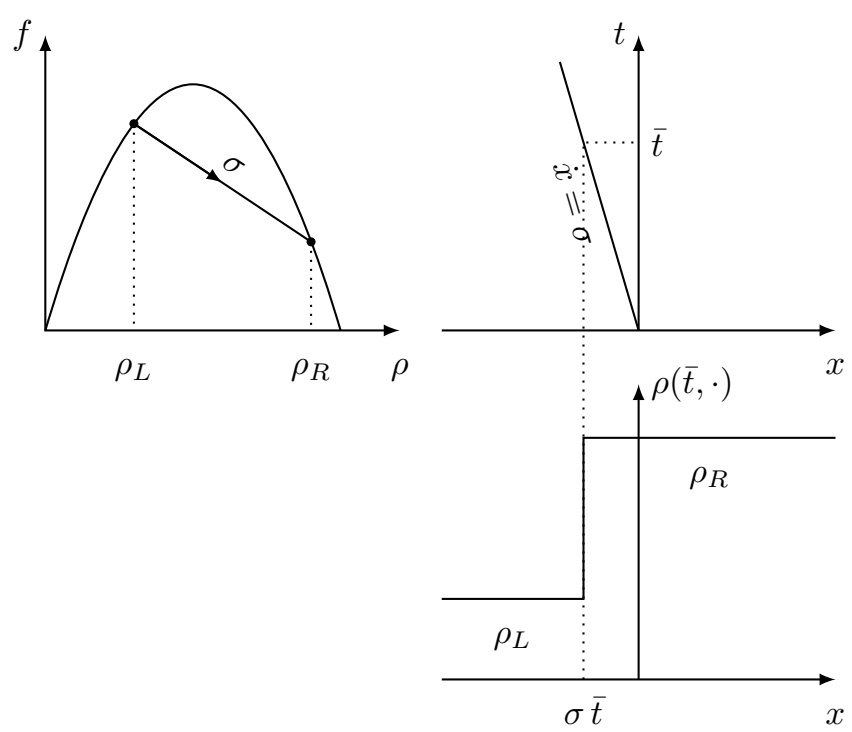

Fig. 9.: A shock wave. On the left we represent the fundamental diagram. On the right we give the solution in the $(x, t)$-plane, above, and its profile $x \mapsto \rho(\bar{t}, x)$ computed at a time $\bar{t}>0$, below.

Summing up, for any $\rho_{L}, \rho_{R} \in\left[0, \rho_{\max }\right]$ Riemann problem (10) admits $\rho(t, x)=\mathcal{R}_{\mathrm{LWR}}\left[\rho_{L}, \rho_{R}\right](x / t)$ as self-similar weak solution, see Fig. 8 and Fig. 9.

The mathematical construction given above for weak self-similar solutions to (10) may seem quite artificial. The following two examples show that such solutions have a clear physical counterpart: an acceleration corresponds to a rarefaction wave and braking produces a shock wave.

Example 5 (Rarefaction wave). Consider cars that are bumper-to-bumper in the upstream of a traffic light placed at $x=0$, which turns from red to green at time $t=0$. The corresponding model is given by Riemann problem (10) with $\rho_{L}=\rho_{\max }$ and $\rho_{R}=0$. The solution is then the rarefaction

$$
\rho(t, x)= \begin{cases}\rho_{\max } & \text { if } x<f^{\prime}\left(\rho_{\max }\right) t \\ \left(f^{\prime}\right)^{-1}(x / t) & \text { if } f^{\prime}\left(\rho_{\max }\right) t \leqslant x \leqslant f^{\prime}(0) t, \\ 0 & \text { if } x>f^{\prime}(0) t .\end{cases}
$$

According to this solution, see Fig. 10, the car initially at $x_{0}<0$ is at rest until time $t_{1}=x_{0} / f^{\prime}\left(\rho_{\max }\right)>0$,

$$
x(t)=x_{0}, \quad t \in\left[0, t_{1}\right],
$$

and then starts to move according to the law

$$
\dot{x}(t)=v(\rho(t, x))=v\left(\left(f^{\prime}\right)^{-1}(x(t) / t)\right), \quad t>t_{1} .
$$
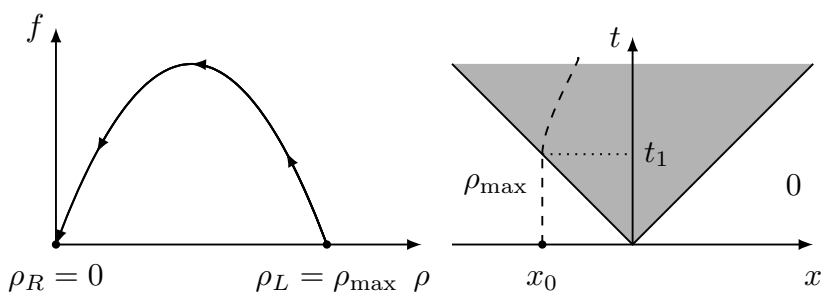

Fig. 10.: The rarefaction considered in Example 5.

The dashed curve corresponds to the trajectory of a car.

Notice that for any $t \geqslant t_{1}$ its trajectory belongs to the cone

$$
f^{\prime}\left(\rho_{\max }\right) t \leqslant x \leqslant f^{\prime}(0) t
$$

simply because $f^{\prime}(0)=v_{\max }$.

This corresponds to what we actually observe in real life: the cars closer to the traffic light start to move earlier and then accelerate.

Example 6 (Shock wave). Consider cars that are equally spaced and moving with the same velocity in the upstream of a traffic light placed at $x=0$, which is red and stays red. This is equivalent to place cars bumperto-bumper in the downstream of the traffic light. The corresponding model is given by Riemann problem (10) with $\rho_{L} \in\left(0, \rho_{\max }\right)$ and $\rho_{R}=\rho_{\max }$. The solution is then the shock wave

$\rho(t, x)=\left\{\begin{array}{ll}\rho_{L} & \text { if } x<\sigma t \\ \rho_{\max } & \text { if } x \geqslant \sigma t\end{array} \quad\right.$ with $\quad \sigma=\frac{f\left(\rho_{L}\right)-f\left(\rho_{\max }\right)}{\rho_{L}-\rho_{\max }}$.

According to this solution, see Fig. 11, the car initially
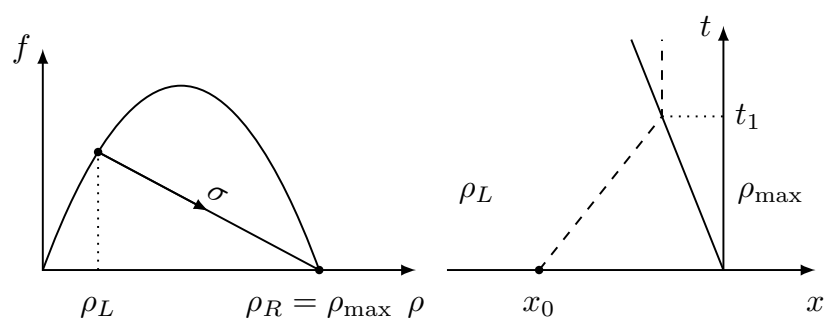

Fig. 11.: The shock considered in Example 6. The dashed curve corresponds to the trajectory of a car.

at $x_{0}<0$ moves with velocity $v=v\left(\rho_{L}\right)>0$ until time $t_{1}=-x_{0} /\left(v\left(\rho_{L}\right)-\sigma\right)>0$,

$$
x(t)=x_{0}+v\left(\rho_{L}\right) t, \quad t \in\left[0, t_{1}\right],
$$


and then stops to move

$$
\dot{x}(t)=0, \quad t>t_{1} .
$$

This corresponds to what we actually observe in real life: once a car reaches the tail of a queue it suddenly stops.

\subsection{Wave-front tracking}

A solution of the Cauchy problem for LWR model (9)

$$
\left\{\begin{array}{l}
\partial_{t} \rho+\partial_{x} f(\rho)=0 \\
\rho(0, x)=\bar{\rho}(x)
\end{array}\right.
$$

can be obtained by applying the wave-front tracking algorithm. Roughly speaking, approximate solutions to (15) are obtained by gluing together approximate solutions to Riemann problems corresponding to the jumps of an approximate initial datum and to the interactions between waves. We give below more details about this construction. For simplicity we assume that $\bar{\rho} \in$ $\mathbf{B V} \cap \mathbf{L}^{\mathbf{1}}\left(\mathbb{R} ;\left[0, \rho_{\max }\right]\right)$.

As a first step we construct an approximate initial datum $\bar{\rho}_{n}$ as follows:

- Fix $n \in \mathbb{N}$ sufficiently large and define in $\left[0, \rho_{\max }\right]$ the mesh

$$
\mathcal{M}_{n}=\left\{\rho_{0}, \rho_{1}, \ldots, \rho_{n}\right\}
$$

where $\rho_{i}=\varepsilon_{n} i$ with $\varepsilon_{n}=\rho_{\max } / n$. Notice that $\rho_{0}=0$ and $\rho_{n}=\rho_{\max }$.

- Approximate the initial datum $\bar{\rho}$ by a piecewise constant function $\bar{\rho}_{n}: \mathbb{R} \rightarrow\left[0, \rho_{\max }\right]$, see Fig. 12 , with a finite number of jumps and such that

$$
\begin{gathered}
\bar{\rho}_{n}(\mathbb{R}) \subseteq \mathcal{M}_{n}, \quad \operatorname{TV}\left(\bar{\rho}_{n}\right) \leqslant \operatorname{TV}(\bar{\rho}), \\
\lim _{n \rightarrow+\infty}\left\|\bar{\rho}_{n}-\bar{\rho}\right\|_{\mathbf{L}^{1}(\mathbb{R})}=0 .
\end{gathered}
$$

The second step is to construct a piecewise constant approximate solution $\rho_{n}: \mathbb{R} \rightarrow \mathcal{M}_{n}$ to the approximate Cauchy problem corresponding to the approximate initial datum $\bar{\rho}_{n}: \mathbb{R} \rightarrow \mathcal{M}_{n}$

$$
\left\{\begin{array}{l}
\partial_{t} \rho+\partial_{x} f(\rho)=0, \\
\rho(0, x)=\bar{\rho}_{n}(x) .
\end{array}\right.
$$

Remark 7. For $t>0$ sufficiently small, the exact solution $\rho_{\mathrm{ex}}$ to $(17)$ can be obtained by gluing the exact solutions of the Riemann problems corresponding to the

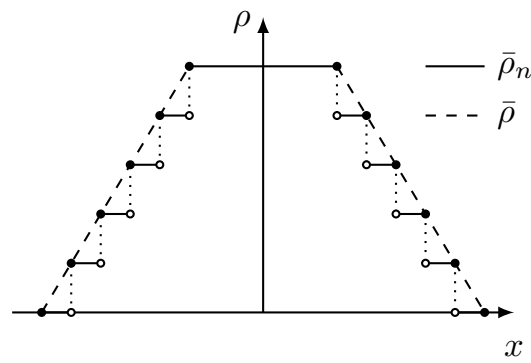

Fig. 12.: An approximate initial datum $\bar{\rho}_{n}$ of the initial datum $\bar{\rho}$.

discontinuity points of $\bar{\rho}_{n}$. This means that if $\bar{\rho}_{n}$ has a discontinuity at $x_{0} \in \mathbb{R}$ and $\lim _{x \rightarrow x_{0}^{ \pm}} \bar{\rho}_{n}=\rho_{0}^{ \pm} \in \mathcal{M}_{n}$, then $\rho_{\mathrm{ex}}(t, x)=\mathcal{R}_{\mathrm{LWR}}\left[\rho_{0}^{-}, \rho_{0}^{+}\right]\left(\left(x-x_{0}\right) / t\right)$ at least for $t>0$ and $\left|x-x_{0}\right|$ sufficiently small. Such construction for $\rho_{\text {ex }}$ holds until an interaction occurs between two waves started from two adjacent discontinuity points of $\bar{\rho}_{n}$. The exact solution $\rho_{\mathrm{ex}}$ can be then prolonged by applying again the Riemann solver $\mathcal{R}_{\mathrm{LWR}}$ at the interaction if and only if $\rho_{\mathrm{ex}}$ is piecewise constant. However this is not in general the case. Indeed $\mathcal{R}_{\mathrm{LWR}}\left[\rho_{L}, \rho_{R}\right]$ is piecewise constant if and only if $\rho_{L} \leqslant \rho_{R}$. On the other hand, if $\rho_{L}>\rho_{R}$ then $\mathcal{R}_{\mathrm{LWR}}\left[\rho_{L}, \rho_{R}\right]$ is a rarefaction and $\mathcal{R}_{\mathrm{LWR}}\left[\rho_{L}, \rho_{R}\right](\mathbb{R})=\left[\rho_{R}, \rho_{L}\right]$.

The considerations in Remark 7 motivate the discretization of the rarefactions and the introduction of the Riemann solver $\mathcal{R}_{\mathrm{LWR}}^{n}: \mathcal{M}_{n}^{2} \rightarrow \mathbf{B V}\left(\mathbb{R} ; \mathcal{M}_{n}\right)$ defined as follows:

(1) If $\rho_{L}, \rho_{R} \in \mathcal{M}_{n}$ are such that $\rho_{L} \leqslant \rho_{R}$, then we define

$$
\mathcal{R}_{\mathrm{LWR}}^{n}\left[\rho_{L}, \rho_{R}\right] \equiv \mathcal{R}_{\mathrm{LWR}}\left[\rho_{L}, \rho_{R}\right] .
$$

(2) If $\rho_{L}, \rho_{R} \in \mathcal{M}_{n}$ are such that $\rho_{L}>\rho_{R}$, then we define

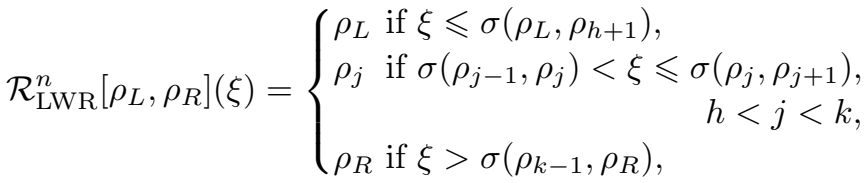

where $\left\{\rho_{h}, \ldots, \rho_{k}\right\}=\left[\rho_{R}, \rho_{L}\right] \cap \mathcal{M}_{n}$ are such that $\rho_{j}>\rho_{j+1}$.

Notice that the discontinuities of the discretized rarefaction satisfy the Rankine-Hugoniot condition (14) and therefore $(t, x) \mapsto \mathcal{R}_{\text {LWR }}^{n}\left[\rho_{L}, \rho_{R}\right](x / t)$ is a weak solution for any $\rho_{L}, \rho_{R} \in\left[0, \rho_{\max }\right]$. 
A piecewise constant approximate solution $\rho_{n}: \mathbb{R} \rightarrow$ $\mathcal{M}_{n}$ to (17) is then obtained by juxtaposing the solutions of the Riemann problems corresponding to the discontinuities of $\bar{\rho}_{n}$ and to the interactions between waves, and solved by applying $\mathcal{R}_{\mathrm{LWR}}^{n}$, see Fig. 13 .
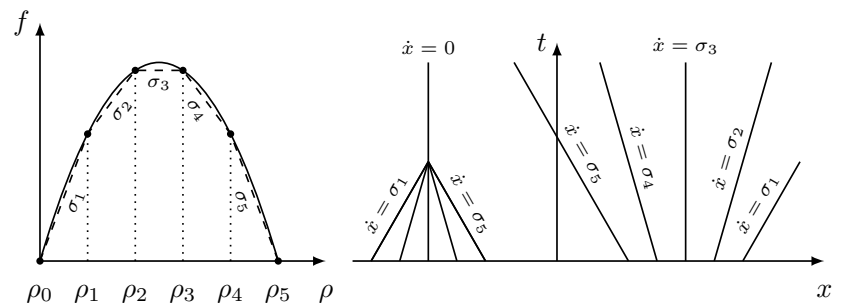

Fig. 13.: Partial construction of a piecewise constant approximate solution $\rho_{n}$ to $(17)$ with initial datum $\bar{\rho}_{n}$ represented in Fig. 12 .

Such procedure can be iterated as long as both the number of waves and the number of interactions are bounded on any bounded time interval; see Fig. 14 for an hypothetical example for which this is not the case.

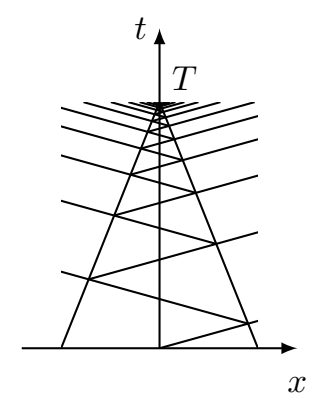

Fig. 14.: An example of infinitely many interactions occurring in a bonded time interval $[0, T]$, see Ref. [36] for its construction.

The global existence of the approximate solution $\rho_{n}$ is ensured by the following proposition.

Proposition 8. After any interaction the number of waves decreases by at least one and the total variation does not increase.

Proof. Consider an interaction between $m \geqslant 2$ waves. Let $\rho_{i}$ and $\rho_{i+1}$ be the left and right states of the $i$-th wave, $i \in\{1, \ldots, m\}$. We distinguish the following cases:
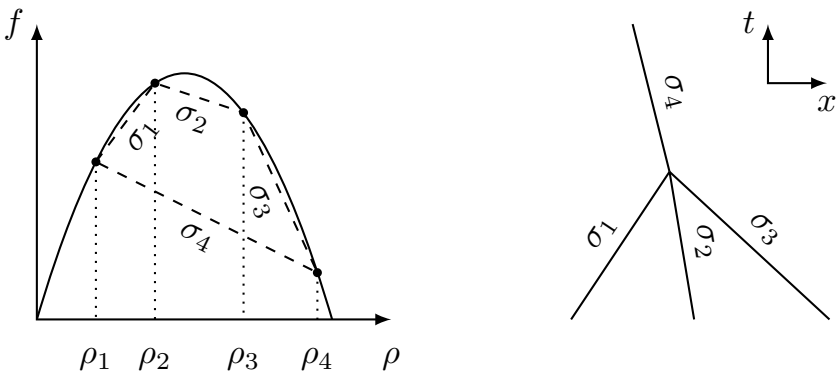

Fig. 15.: An interaction involving three shock waves separating $\rho_{1}$ and $\rho_{2}, \rho_{2}$ and $\rho_{3}$, and $\rho_{3}$ and $\rho_{4}$. On the left we represent in the fundamental diagram both the states, $\rho_{i}$, and the speeds of propagation, $\sigma_{i}$. Indeed, for instance, $\sigma_{1}=\sigma\left(\rho_{1}, \rho_{2}\right)$ is the slope of the segment joining $\left(\rho_{1}, f\left(\rho_{1}\right)\right)$ to $\left(\rho_{2}, f\left(\rho_{2}\right)\right)$, as well as the speed of propagation of the shock wave separating the states $\rho_{1}$ and $\rho_{2}$. On the right we represent the shock waves in the $(x, t)$-plane.
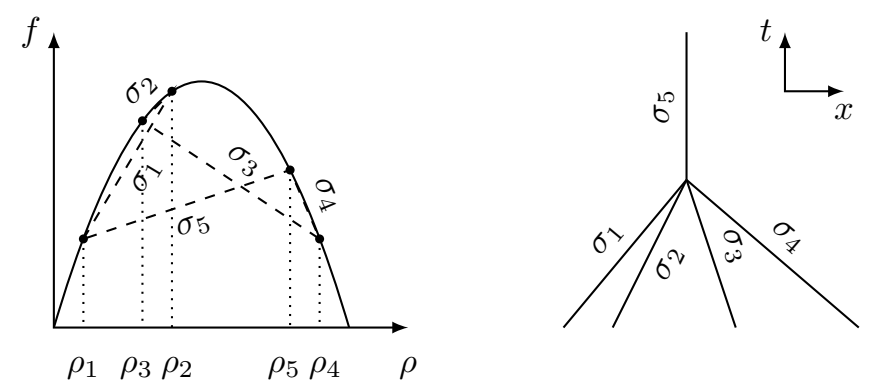

Fig. 16.: An interaction involving four waves separating $\rho_{1}$ and $\rho_{2}, \rho_{2}$ and $\rho_{3}, \rho_{3}$ and $\rho_{4}$, and $\rho_{4}$ and $\rho_{5}$. On the left we represent in the fundamental diagram both the states, $\rho_{i}$, and the speeds of propagation, $\sigma_{i}$. Indeed, for instance, $\sigma_{1}=\sigma\left(\rho_{1}, \rho_{2}\right)$ is the slope of the segment joining $\left(\rho_{1}, f\left(\rho_{1}\right)\right)$ to $\left(\rho_{2}, f\left(\rho_{2}\right)\right)$, as well as the speed of propagation of the shock wave separating the states $\rho_{1}$ and $\rho_{2}$. On the right we represent the shock waves in the $(x, t)$ plane.

(1) Assume that all the involved waves are shock waves, see Fig. 15. In this case $\rho_{i}<\rho_{i+1}$ and the result of the interaction is a single shock wave between $\rho_{1}$ and $\rho_{m+1}$. Therefore the number of waves decreases by at least one and the total variation does not change.

(2) Assume that also rarefaction waves are involved in 
February 25, 2020 16:29 Preprint-9×6 Book Title: Order, Disorder and Criticality: Advanced Problems of Preprint page 12 Phase Transition Theory. Ed. by Yu. Holovatch. Vol. 6, 2020, World Scientific, Singapore

the interaction, see Fig. 16. If the $i$-th wave is a rarefaction wave, then $\rho_{i}-\rho_{i+1}=\varepsilon_{n}$; moreover if $i>1$ then the $(i-1)$-th wave is a shock wave with $\rho_{i}-\rho_{i-1} \geqslant 2 \varepsilon_{n}$ and if $i<m$ then the $(i+1)$-th wave is a shock wave with $\rho_{i+2}-\rho_{i+1} \geqslant 2 \varepsilon_{n}$. As in the previous case the result of the interaction is a single shock wave between $\rho_{1}$ and $\rho_{m+1}$, the number of waves decreases by at least one and, differently from the previous case, the total variation strictly decreases.

Since the above cases encompasses all the possible interactions, the proof is complete.

By Proposition 8 the approximate solution $\rho_{n}$ can be constructed globally in time. Moreover if $t_{h}<t_{h+1}$ are two consecutive interaction times, then for any $t \in\left(t_{h}, t_{h+1}\right)$ the approximate solution $\rho_{n}(t, \cdot)$ takes the form

$$
\begin{aligned}
\rho_{n}(t, x)= & \rho_{\frac{1}{2}}^{h} \cdot \mathbb{1}_{\left(-\infty, x_{1}^{h}(t)\right)}(t) \\
& +\sum_{i=1}^{\mathrm{N}^{h}-1} \rho_{i+\frac{1}{2}}^{h} \cdot \mathbb{1}_{\left[x_{i}^{h}(t), x_{i+1}^{h}(t)\right)}(t) \\
& +\rho_{\mathrm{N}^{h}+\frac{1}{2}}^{h} \cdot \mathbb{1}_{\left[x_{\mathrm{N}}^{h}(t),+\infty\right)}(t),
\end{aligned}
$$

where $\mathbb{1}_{I}$ is the indicator function of the interval $I \subset \mathbb{R}$, $\rho_{\frac{1}{2}}^{h}, \ldots, \rho_{N^{h}+\frac{1}{2}}^{h} \in \mathcal{M}_{n}$ are constant with $\rho_{i-\frac{1}{2}}^{h} \neq \rho_{i+\frac{1}{2}}^{h}$, $x_{i}^{h}(t) \in \mathbb{R}$ is a discontinuity point of $\rho_{n}(t, \cdot)$ with $\dot{x}_{i}^{h}(t)=$ $\sigma\left(\rho_{i-\frac{1}{2}}^{h}, \rho_{i+\frac{1}{2}}^{h}\right)$, and $\mathbb{N}^{h}=N^{h}(t) \in \mathbb{N}$ is the number of discontinuity points of $\rho_{n}(t, \cdot)$. We stress that $t \mapsto N^{h}(t)$ is piecewise constant and non-increasing by Proposition 8 .

The next step is to prove that (up to a subsequence) $\left\{\rho_{n}\right\}_{n}$ converges in $\mathbf{L}_{\text {loc }}^{\mathbf{1}}([0,+\infty) \times \mathbb{R})$. To do so we need the estimates provided by the following proposition.

Proposition 9. There exists a constant $C>0$ such that for any $t, s \geqslant 0$ and $n \in \mathbb{N}$ we have

$$
\begin{gathered}
\operatorname{TV}\left(\rho_{n}(t, \cdot)\right) \leqslant \operatorname{TV}(\bar{\rho}), \quad\left\|\rho_{n}(t, \cdot)\right\|_{\mathbf{L}^{\infty}(\mathbb{R})} \leqslant \rho_{\max }, \\
\left\|\rho_{n}(t, \cdot)-\rho_{n}(s, \cdot)\right\|_{\mathbf{L}^{\mathbf{1}}(\mathbb{R})} \leqslant C|t-s| .
\end{gathered}
$$

Proof. The first estimate follows from Proposition 8 and $(16)_{2}$. By construction $\rho_{n}$ takes values in $\mathcal{M}_{n} \subset\left[0, \rho_{\max }\right]$, hence the second estimate holds true. The last estimate follows from the fact that $f$ is Lipschitz continuous and its Lipschitz constant $L=\sup _{\left[0, \rho_{\max }\right]}\left|f^{\prime}\right|$ bounds the speed of propagation of any wave. Let's

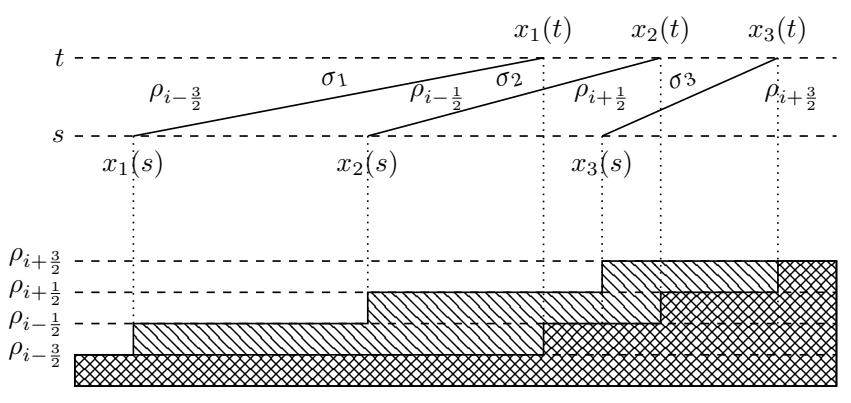

Fig. 17.: The profiles of $\rho_{n}(t, \cdot)$ and $\rho_{n}(s, \cdot)$ in the case no interaction occurs in the time interval $[s, t]$.

prove it. Let $t_{h}<t_{h+1}$ be two consecutive interaction times. Then $\rho_{n}$ takes the form (18) in the time interval $\left(t_{h}, t_{h+1}\right)$. Fix $s, t \in\left(t_{h}, t_{h+1}\right)$. Notice that $x_{i}^{h}(t)=x_{i}^{h}(s)+\sigma\left(\rho_{i-\frac{1}{2}}^{h}(t), \rho_{i+\frac{1}{2}}^{h}(t)\right) \cdot(t-s)$ for any $i \in\left[1, \mathrm{~N}^{h}\right] \cap \mathbb{N}$. Hence, see Fig. 17, we have

$$
\begin{array}{r}
\left\|\rho_{n}(t, \cdot)-\rho_{n}(s, \cdot)\right\|_{\mathbf{L}^{1}(\mathbb{R})}= \\
\sum_{i=1}^{\mathrm{N}^{h}}\left|(t-s) \cdot \sigma\left(\rho_{i-\frac{1}{2}}^{h}-\rho_{i+\frac{1}{2}}^{h}\right) \cdot\left(\rho_{i-\frac{1}{2}}^{h}-\rho_{i+\frac{1}{2}}^{h}\right)\right| \leqslant \\
L \cdot|t-s| \cdot \sum_{i=1}^{\mathrm{N}^{h}}\left|\rho_{i-\frac{1}{2}}^{h}-\rho_{i+\frac{1}{2}}^{h}\right|= \\
L \cdot|t-s| \cdot \operatorname{TV}\left(\rho_{n}(t, \cdot)\right) \leqslant L \cdot|t-s| \cdot \operatorname{TV}(\bar{\rho}) .
\end{array}
$$

The case when one or more interactions take place for times between $t$ and $s$ is similar, because by the finite speed of propagation of the waves the map $t \mapsto \rho_{n}(t, \cdot)$ is $\mathbf{L}^{\mathbf{1}}$-continuous across interaction times. Therefore it is sufficient to take $C=\operatorname{TV}(\bar{\rho}) \cdot L$.

By (19) we can apply Helly's theorem in the form [33, Theorem 2.4], and obtain that (up to a subsequence) $\left\{\rho_{n}\right\}_{n}$ converges in $\mathbf{L}_{\text {loc }}^{\mathbf{1}}([0,+\infty) \times \mathbb{R})$ to a function $\rho$ and that

$$
\begin{gathered}
\operatorname{TV}(\rho(t, \cdot)) \leqslant \operatorname{TV}(\bar{\rho}), \quad\|\rho(t, \cdot)\|_{\mathbf{L}^{\infty}(\mathbb{R})} \leqslant \rho_{\max }, \\
\|\rho(t, \cdot)-\rho(s, \cdot)\|_{\mathbf{L}^{\mathbf{1}}(\mathbb{R})} \leqslant C|t-s| .
\end{gathered}
$$

At last we have to prove that $\rho$ is actually a weak solution of the original Cauchy problem (15).

Proposition 10. $\rho$ is a weak solution of Cauchy problem (15), namely $\rho\left(0^{+}, x\right)=\bar{\rho}(x)$ for almost every $x \in \mathbb{R}$ and

$$
\begin{aligned}
\int_{0}^{+\infty} \int_{\mathbb{R}}\left(\rho \partial_{t} \varphi+f(\rho) \partial_{x} \varphi\right) \mathrm{d} x \mathrm{~d} t=0 \\
\forall \varphi \in \mathbf{C}_{\mathbf{c}}^{\infty}((0,+\infty) \times \mathbb{R} ; \mathbb{R})
\end{aligned}
$$


Proof. The initial condition is satisfied almost everywhere because, by $(16)_{3},(19)_{3}$ and the $\mathbf{L}_{\mathbf{l o c}}^{\mathbf{1}}$-convergence of $\rho_{n}$ to $\rho$, for any $a<b$ we have that

$$
\begin{array}{r}
\int_{a}^{b}|\rho(t, x)-\bar{\rho}(x)| \mathrm{d} x \leqslant \\
\int_{a}^{b}\left|\rho(t, x)-\rho_{n}(t, x)\right| \mathrm{d} x+\int_{a}^{b}\left|\rho_{n}(t, x)-\bar{\rho}_{n}(x)\right| \mathrm{d} x \\
+\int_{a}^{b}\left|\bar{\rho}_{n}(x)-\bar{\rho}(x)\right| \mathrm{d} x \underset{n \rightarrow 0^{+}}{\rightarrow} 0 .
\end{array}
$$

Take $\varphi$ in $\mathbf{C}_{\mathbf{c}}^{\infty}((0,+\infty) \times \mathbb{R} ; \mathbb{R})$. Choose $T>0$ such that $\varphi(t, \cdot) \equiv 0$ for any $t \geqslant T$. Since $\rho_{n}$ is uniformly bounded and $f$ is uniformly continuous on bounded sets, it is sufficient to prove that

$$
\int_{0}^{T} \int_{\mathbb{R}}\left(\rho_{n} \partial_{t} \varphi+f\left(\rho_{n}\right) \partial_{x} \varphi\right) \mathrm{d} x \mathrm{~d} t \underset{n \rightarrow+\infty}{\longrightarrow} 0 .
$$

By (18) the approximate solution $\rho_{n}(t, \cdot)$ has a jump at $x_{1}(t), \ldots, x_{N(t)}(t)$ with $x_{i}(t)<x_{i+1}(t)$. The polygonal lines $x=x_{i}(t)$ subdivide the strip $[0, T] \times \mathbb{R}$ into finitely many regions $\Gamma_{i}$ where $\rho_{n}$ is constant. Introducing the vector

$$
\Phi \equiv\left(\varphi \rho_{n}, \varphi f\left(\rho_{n}\right)\right),
$$

by the Gauss-Green formula the double integral in (20) can be written as

$$
\sum_{i} \iint_{\Gamma_{i}} \operatorname{div} \Phi(t, x) \mathrm{d} x \mathrm{~d} t=\sum_{i} \int_{\partial \Gamma_{i}} \Phi \cdot \boldsymbol{n} \mathrm{d} s .
$$

Above $\partial \Gamma_{i}$ is the oriented boundary of $\Gamma_{i}$, while $\boldsymbol{n}$ is the outer unit normal vector. Observe that $\boldsymbol{n} \mathrm{d} s=$ $\pm\left(\dot{x}_{i},-1\right) \mathrm{d} t$ along each polygonal line $x=x_{i}(t)$, while $\varphi(t, x)=0$ along the lines $t=0$ and $T=0$. By (21) the double integral in (20) is equal to

$\int_{0}^{T} \sum_{i}\left(\dot{x}_{i}\left(\rho_{i+\frac{1}{2}}-\rho_{i-\frac{1}{2}}\right)-\left(f\left(\rho_{i+\frac{1}{2}}\right)-f\left(\rho_{i-\frac{1}{2}}\right)\right)\right) \varphi\left(t, x_{i}(t)\right) \mathrm{d} t$. By construction $\dot{x}_{i}=\sigma\left(\rho_{i+\frac{1}{2}}, \rho_{i+\frac{1}{2}}\right)$, hence

$$
\dot{x}_{i}\left(\rho_{i+\frac{1}{2}}-\rho_{i-\frac{1}{2}}\right)-\left(f\left(\rho_{i+\frac{1}{2}}\right)-f\left(\rho_{i-\frac{1}{2}}\right)\right)=0
$$

and therefore (20) is trivial.

It can be proved that $\rho$ is not the only weak solution of Cauchy problem (15), but it is the unique weak solution satisfying the Kruzhkov ${ }^{37,38}$ condition

$$
\begin{array}{r}
\int_{\mathbb{R}} \int_{0}^{+\infty}\left(|\rho(t, x)-k| \partial_{t} \varphi(t, x)+F(\rho(t, x), k) \partial_{x} \varphi(t, x)\right) \mathrm{d} t \mathrm{~d} x \geqslant 0 \\
\forall \varphi \in \mathbf{C}_{\mathbf{c}}^{\infty}((0,+\infty) \times \mathbb{R} ;[0,+\infty)), k \in\left[0, \rho_{\max }\right],
\end{array}
$$

where

$$
F(\rho, k)=\operatorname{sign}(\rho-k)[f(\rho)-f(k)] .
$$

In poor words, Kruzhkov condition (22) selects the only weak solution with only physically reasonable shocks, that are the discontinuities across which the density increases. Indeed only braking produces a shock wave and only an increase of the density produces braking, therefore the density has to increase across a shock wave. We recall that Kruzhkov condition (22) can be deduced by applying the vanishing viscosity method, namely by considering the solutions $\rho_{\varepsilon}$ of the viscous approximations

$$
\partial_{t} \rho+\partial_{x} f(\rho)=\varepsilon \partial_{x}^{2} \rho, \quad \varepsilon>0,
$$

which converge to $\rho$ as $\varepsilon$ goes to zero. Furthermore in Ref. [37] the uniqueness is proved by applying the doubling of variables method.

\section{Links between microscopic and macro- scopic models}

In this section we give some basic ideas on how it is possible to derive the first order microscopic FTL model (6) from the scalar macroscopic LWR model (9) and viceversa.

We start by showing how it is possible to heuristically derive a first order FTL model from the LWR model:

- Let $\rho$ be the unique weak solution of LWR model (9) satisfying Kruzhkov condition (22).

- Let $F$ be the cumulative distribution of $\rho$

$$
F(t, x)=\int_{-\infty}^{x} \rho(t, y) \mathrm{d} y .
$$

- Let $X$ be the pseudo inverse of $F$

$$
X(t, z)=\inf \{x \in \mathbb{R}: F(x)>z\}, \quad z \in[0, L] .
$$

- Formally, $X(t, z)$ satisfies $F(t, X(t, z))=z$ and therefore

$$
\begin{aligned}
& \left\{\begin{array}{l}
\partial_{x} F=\rho \\
\partial_{t} F=-\rho v(\rho)
\end{array}\right. \\
\Rightarrow & \left\{\begin{array}{l}
1=\partial_{z} F(t, X(t, z))=\left(\partial_{x} F\right)\left(\partial_{z} X\right)=\rho\left(\partial_{z} X\right) \\
0=\partial_{t} F(t, X(t, z))=\partial_{t} F+\left(\partial_{x} F\right)\left(\partial_{t} X\right)=\rho\left(\partial_{t} X-v(\rho)\right)
\end{array}\right. \\
\Rightarrow & X_{t}=v\left(1 / X_{z}\right) .
\end{aligned}
$$

- Approximating the obtained equation via a forward $z$-finite difference with step $\ell$ gives

$$
\begin{aligned}
X_{t}(t, z)=v\left(\frac{\ell}{X(t, z+\ell)-X(t, z)}\right), \\
z \in\{0, \ldots,(n-1) \ell\} .
\end{aligned}
$$


The obtained system corresponds to the first order FTL model (6), which is therefore a discrete Lagrangian version of $(9)$.

Conversely, below we show the main steps to approximate the unique weak solution of the Cauchy problem for LWR model (15) satisfying Kruzhkov condition (22) via a particle system governed by the first order FTL model (6):

(1) Fix an initial datum $\rho_{0}$ and let $L=\left\|\rho_{0}\right\|_{\mathbf{L}^{\mathbf{1}}(\mathbb{R})}$ be the total length of the traffic.

(2) Split $\bar{\rho}$ in $n \in \mathbb{N}$ platoons of (possible fractional) cars with length $\ell=L / n$ and with (ordered) end points

$$
\bar{x}_{n}<\bar{x}_{n-1}<\ldots<\bar{x}_{1}<\bar{x}_{0} .
$$

(3) "Particles" with mass $\ell$ are initially placed in $\bar{x}_{0}, \ldots, \bar{x}_{n}$ and evolve according to the first order microscopic FTL model

$$
\begin{cases}\dot{x}_{0}(t)=v_{\max }, & \\ \dot{x}_{i}(t)=v\left(\frac{\ell}{x_{i-1}(t)-x_{i}(t)}\right), & i \in\{1, \ldots, n\}, \\ x_{i}(0)=\bar{x}_{i}, & i \in\{0, \ldots, n\} .\end{cases}
$$

(4) Introduce the discrete density

$$
\rho_{n}(t, x)=\sum_{i=1}^{n} \frac{\ell}{x_{i}(t)-x_{i-1}(t)} \mathbb{1}_{\left[x_{i-1}(t), x_{i}(t)\right)}(x) .
$$

We have then the following result.

Theorem 11. Let $\rho$ be the unique weak solution of the Cauchy problem for LWR model (15)

$$
\left\{\begin{array}{l}
\partial_{t} \rho+\partial_{x} f(\rho)=0 \\
\rho(0, x)=\bar{\rho}(x)
\end{array}\right.
$$

satisfying Kruzhkov condition (22). Assume that $\bar{\rho}$ is in $\mathbf{L}^{\mathbf{1}} \cap \mathbf{L}^{\infty}(\mathbb{R})$, and $v$ is in $\mathbf{C}^{\mathbf{1}}([0,+\infty) ; \mathbb{R})$ and is strictly decreasing. If one of the following conditions is satisfied

$$
\begin{gathered}
\bar{\rho} \in \mathbf{B V}(\mathbb{R}), \\
\rho \mapsto \rho v^{\prime}(\rho) \text { is non-increasing, }
\end{gathered}
$$

then (up to a subsequence) $\left\{\rho_{n}\right\}_{n}$ given by (24) converges to $\rho$ almost everywhere and in $\mathbf{L}_{\text {loc }}^{\mathbf{1}}$ on $\mathbb{R}_{+} \times \mathbb{R}$.

Proof. Below we give only the main ideas of the proof. The rigorous proof is quite technical and can be found in Ref. [14,39,40].
(1) Prove by contradiction a maximum principle which ensures that the particles preserve their order and the global existence for the solution of FTL model (6).

(2) Prove by induction a uniform BV estimate for $\rho_{n}(t, \cdot)$.

(a) If $\bar{\rho}$ is in $\mathbf{B V}$, then prove a uniform $\mathbf{B V}$ contraction property for $\rho_{n}(t, \cdot)$.

(b) If $\rho \mapsto \rho v^{\prime}(\rho)$ is non-increasing, then prove a uniform discrete Oleinik condition for $\rho_{n}(t, \cdot)$.

(3) Prove that $\left\{\rho_{n}\right\}_{n}$ converges to a measure $\rho$ in $\mathbf{L}_{\text {loc }}^{\mathbf{1}}\left(\overline{\mathbb{R}}_{+} ; d_{L, 1}\right)$, where $d_{L, 1}$ is the rescaled Wasserstein distance defined by

$$
d_{L, 1}\left(\rho_{1}, \rho_{2}\right)=\left\|F_{\rho_{1}}-F_{\rho_{2}}\right\|_{\mathbf{L}^{1}(\mathbb{R} ; \mathbb{R})}
$$

for any non-negative Radon measures $\rho_{1}, \rho_{2}$ in $\mathbb{R}$ with compact support and such that $\rho_{1}(\mathbb{R})=L=\rho_{2}(\mathbb{R})$, where $F_{\rho_{i}}(x)=\rho_{i}((-\infty, x]), i \in\{1,2\}$.

(a) Prove a continuity estimate for $\rho_{n}$ in $\mathbf{L}_{\mathbf{l o c}}^{\mathbf{1}}\left(\overline{\mathbb{R}}_{+} ; d_{L, 1}\right)$ uniform in $t$ and $n$, namely for $X_{n}$ in $\mathbf{L}_{\text {loc }}^{\mathbf{1}}\left(\overline{\mathbb{R}}_{+} \times[0, L]\right)$.

(b) Apply a generalised Aubin-Lions lemma.

(4) Prove that $\rho_{n}$ is a weak solution and then use the strong $\mathbf{L}^{\mathbf{1}}$-compactness to pass to the limit and get that $\rho$ is a weak solution.

(5) Prove that $\rho$ satisfy Kruzhkov condition (22) by carefully estimating several terms appearing after introducing $\rho_{n}$ in (22).

For completeness we recall the generalised AubinLions lemma.

\section{Theorem 12 (Generalized Aubin-Lions lemma).} Let $T, L>0$ be fixed constants and $I \subset \mathbb{R}$ be a bounded open interval. Assume $w: \mathbb{R} \rightarrow \mathbb{R}$ is a Lipschitz continuous and strictly monotone function. Let $\left\{\rho_{n}\right\}_{n \in \mathbb{N}}$ be a sequence in $\mathbf{L}^{\infty}((0, T) \times \mathbb{R}) \cap \mathcal{M}_{L}$ such that:

(1) $\rho_{n}:(0, T) \rightarrow \mathbf{L}^{\mathbf{1}}(\mathbb{R})$ is measurable for all $n \in \mathbb{N}$;

(2) $\operatorname{spt}\left(\rho_{n}(t)\right) \subseteq I$ for all $t \in(0, T)$ and $n \in \mathbb{N}$;

(3) $\sup _{n \in \mathbb{N}} \int_{0}^{T}\left(\left\|w\left(\rho_{n}(t)\right)\right\|_{\mathbf{L}^{1}(I)}+\operatorname{TV}\left(w\left(\rho_{n}(t)\right)\right)\right) \mathrm{d} t<+\infty$;

(4) there exists a constant $C$ depending only on $T$ such that

$d_{L, 1}\left(\rho_{n}(s), \rho_{n}(t)\right) \leq C|t-s| \quad \forall s, t \in(0, T), \forall n \in \mathbb{N}$. 
Then, $\left\{\rho_{n}\right\}_{n \in \mathbb{N}}$ is strongly relatively compact in $\mathbf{L}^{\mathbf{1}}((0, T) \times \mathbb{R})$.

The importance of the micro-macro limit described above is twofold: first, it justifies at the microscopic level the use of macroscopic models; second, it allows to develop macroscopic models on the basis of microscopic interaction rules. In particular, this procedure leads to select a unique Riemann solver for a macroscopic model, which is not necessarily the classical Kruzhkov Riemann solver; see for instance Ref. [14,17,18,39-59] for macroscopic models justified or developed on the basis of this procedure.

\section{Heterogeneous traffic}

We motivate the need to introduce new models by listing the main drawbacks of both FTL model (6) and LWR model (9):

- Traffic is typically heterogeneous: assuming that all cars have the same length and maximal velocity is sometimes a severe restriction.

- Unrealistic behavior of the drivers, that adjust instantaneously their velocities according to the densities they are experiencing.

- Experimental data show that the fundamental diagram $(\rho, \rho v)$ is given by a cloud of points rather than the support of a map $\rho \mapsto \rho v(\rho)$.

- They do not reproduce the amplification of small perturbations (phantom traffic jams), due to the so called "over reaction".

- They do not reproduce hysteresis processes (acceleration and deceleration follow two different branches in the density-flow diagram).

In this section we generalize both models (6) and (9) to the case of an heterogeneous traffic, mainly given by trucks and cars, see Fig. 18. We characterize each ve-

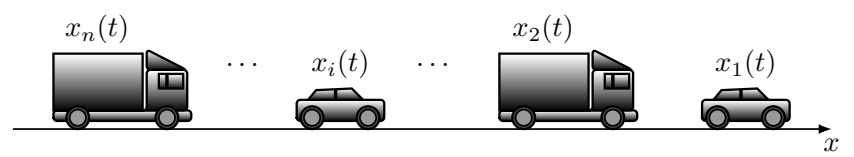

Fig. 18.: An heterogeneous vehicle traffic.

hicle by its length and maximal speed. Since trucks are longer and (typically) slower than cars, we can assume that longer vehicles are slower. Moreover, it is reasonable to assume that length and maximal speed of a vehicle do not change in time.

We encode this information (maximal speed and a length) by expressing the velocity $v$ in terms of Lagrangian marker $w$ and density $\rho$ as follows

$$
v(\rho, w)=w-p(\rho),
$$

with $p(\rho)=\rho^{\gamma}$, where $\gamma>0$ is a parameter of the model. Here $p$ plays the role of an anticipation factor, taking into account divers' reactions to the state of traffic in front of them.

Remark 13. In general it is sufficient to assume for $p:\left[0, \rho_{\max }\right] \rightarrow \mathbb{R}$ that

- $p(0)=0$,

- $p^{\prime}(\rho)>0$ and $2 p^{\prime}(\rho)+p^{\prime \prime}(\rho) \rho>0$ for every $\rho>0$.

The choice $p(\rho)=\rho^{\gamma}, \gamma>0$, is made here only to avoid technicalities linked to working in a general framework. At the same time, we avoid to use the explicit expression of $p$ whenever it is not necessary.

Equation (25) means that a vehicle characterized by Lagrangian marker $w$ moves with velocity $v=w-p(\rho)$ if it experiences the density $\rho$. As a consequence, a vehicle with Lagrangian marker $w$ has maximal speed $v(0, w)=w$. Moreover, since a vehicle is at rest if and only if it is "bumper-to-bumper", the length $\ell$ of a vehicle with Lagrangian marker $w$ satisfies $v(1 / \ell, w)=0$ and therefore

$$
\ell=1 / p^{-1}(w) .
$$

Notice that the length $\ell$ is lower for higher values of the maximal speed $w$.

For simplicity in the exposition we consider separately the microscopic and the macroscopic models.

\subsection{Microscopic model}

By introducing the velocity function (25) in the first order FTL model (6) we obtain

$$
\begin{cases}\dot{x}_{1}(t)=w_{1}, & \\ \dot{x}_{i}(t)=v\left(\frac{1}{x_{i-1}(t)-x_{i}(t)}, w_{i}\right), & i \in\{2, \ldots, n\}, \\ x_{i}(0)=\bar{x}_{i}, & i \in\{1, \ldots, n\},\end{cases}
$$


where $w_{i}$ is the maximal speed of the $i$-th vehicle. System (26) can be written as the Cauchy problem for a system of second order non-linear ODEs

$$
\left\{\begin{array}{rr}
\dot{x}_{i}(t)=v_{i}(t), & i \in\{1, \ldots, n\}, \\
\dot{v}_{i}(t)=p^{\prime}\left(\frac{1}{x_{i-1}(t)-x_{i}(t)}\right) \frac{v_{i-1}(t)-v_{i}(t)}{\left(x_{i-1}(t)-x_{i}(t)\right)^{2}}, & i \in\{2, \ldots, n\}, \\
x_{i}(0)=\bar{x}_{i}, & i \in\{1, \ldots, n\}, \\
v_{1}(0)=w_{1}, & \\
v_{i}(0)=v\left(\frac{1}{\bar{x}_{i-1}-\bar{x}_{i}}, w_{i}\right), &
\end{array}\right.
$$

The above model is called second order FTL model.

\subsection{Macroscopic model}

By introducing the velocity function (25) in the conservation law (8) we obtain

$$
\partial_{t} \rho+\partial_{x}(\rho v(\rho, w))=0 .
$$

Since we have two unknown variables, $\rho$ and $w$, we need a second PDE. By assumption the maximal speed and length of each vehicle do not change in time. This implies that $w$ is constant along any trajectory $t \mapsto x(t)$ and therefore

$$
\frac{\mathrm{d}}{\mathrm{d} t} w(t, x(t))=\partial_{t} w+\dot{x} \partial_{x} w=\partial_{t} w+v(\rho, w) \partial_{x} w=0 .
$$

We just obtained the transport equation

$$
\partial_{t} w+v(\rho, w) \partial_{x} w=0 .
$$

By coupling the conservation law (27) together with the transport equation (28) we obtain the $2 \times 2$ system of PDEs

$$
\left\{\begin{array}{l}
\partial_{t} \rho+\partial_{x}(\rho v)=0, \\
\partial_{t} w+v \partial_{x} w=0 .
\end{array}\right.
$$

This is a second order macroscopic model and is the AwRascle-Zhang (ARZ) model. ${ }^{15,16}$

ARZ model can be interpreted as a generalization of the LWR model to a heterogeneous traffic. In poor words, according to LWR model $(\rho, f)$ belongs to a unique fundamental diagram $\rho \mapsto f(\rho)=\rho v(\rho)$, whereas ARZ model allows to consider a one parameter family of fundamental diagrams $\rho \mapsto f_{w}(\rho)=f_{w}(\rho)=\rho v(\rho, w)$, where $v(\rho, w)=w-p(\rho)$. Notice that if we consider only one type of vechicle, then $w$ is constant and ARZ model (29) reduces to LWR model (9) with velocity function given by $v(\rho)=w-p(\rho)$, which satisfies $(\mathrm{V})$.

As for LWR model, we construct a Riemann solver for ARZ model. Consider the Riemann problem for ARZ model (29)

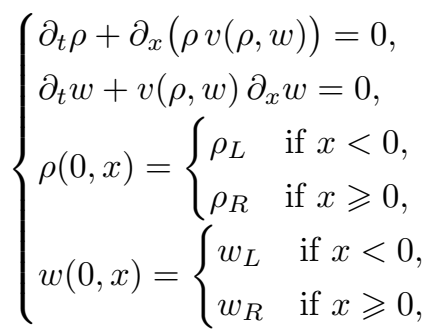

where $W_{L}=\left(\rho_{L}, w_{L}\right), W_{R}=\left(\rho_{R}, w_{R}\right) \in \Omega$ are constant, and $\Omega$ is the physical domain defined by

$$
\Omega=\{(\rho, w) \in[0,+\infty) \times(0,+\infty): w \geqslant p(\rho)\} .
$$

Notice that $v(W) \geqslant 0$ for any $W=(\rho, w)$ belonging to $\Omega$.

We need to distinguish the following cases to construct a solution to $(30)$.

- If $W_{L}=W_{R}$, then the constant function $(\rho, w) \equiv$ $W_{L, R}$ is the solution.

- If $w_{L}=w_{R}$, then all the vehicles have the same length and maximal speed, hence ARZ model reduces to LWR model. Indeed, introducing $\left(\rho, w_{L, R}\right)$ in (30) we obtain (10) with $f(\rho)=\rho\left(w_{L, R}-\right.$ $p(\rho))$. For what we have already seen in Section 3.1, the solution to (30) is then given by $W(t, x)=$ $\left(\mathcal{R}_{\mathrm{LWR}}\left[\rho_{L}, \rho_{R}\right](x / t), w_{L, R}\right)$ and it can be either a rarefaction (if $\rho_{L}>\rho_{R}$ ) or a shock (if $\rho_{L}<\rho_{R}$ ).

- If $w_{L} \neq w_{R}$, then we have two families of vehicles, one in $(-\infty, 0)$ and one in $[0,+\infty)$. By assumption overtaking is not allowed, hence the two families do not mix each other. The vehicles are anisotropic (respond only to frontal stimuli), hence the vehicles with Lagrangian marker $w_{R}$ are not influenced by the other vehicles; for this reason they move with speed $v_{R}=v\left(W_{R}\right)=w_{R}-p\left(\rho_{R}\right)$. As a consequence the $w$-component of the solution to $(30)$ is

$$
w(t, x)= \begin{cases}w_{L} & \text { if } x<v_{R} t, \\ w_{R} & \text { if } x \geqslant v_{R} t .\end{cases}
$$

In $x<v_{R} t$ we have only vehicles with Lagrangian marker $w_{L}$ and therefore their dynamics can be described by the LWR model. Assuming that each of 
them moves with speed $v_{L}=w_{L}-p\left(\rho_{L}\right)$ may lead to a contradiction because their velocity cannot exceed $v_{R}$ (overtaking is not allowed). Also assuming that each of them moves with speed $v_{R}$ may lead to a contradiction because their velocity cannot exceed their maximal speed $w_{L}$. For this reason we introduce the intermediate density $\widetilde{\rho} \in\left[0, p^{-1}\left(w_{L}\right)\right]$ implicitly defined by

$$
v\left(\widetilde{\rho}, w_{L}\right)=\min \left\{w_{L}, v_{R}\right\}
$$

and take

$$
\rho(t, x)= \begin{cases}\mathcal{R}_{\mathrm{LWR}}\left[\rho_{L}, \widetilde{\rho}\right](x / t) & \text { if } x<v_{R} t \\ \rho_{R} & \text { if } x \geqslant v_{R} t\end{cases}
$$

as $\rho$-component of the solution to (30).

The above construction leads to the following definition.

Definition 14. The Riemann solver $\mathcal{R}_{\mathrm{ARZ}}: \Omega^{2} \rightarrow$ $\mathbf{B V}(\mathbb{R} ; \Omega)$ associated to ARZ model (29) is defined by

$$
\mathcal{R}_{\mathrm{ARZ}}\left[W_{L}, W_{R}\right] \equiv\left(\phi_{1}, \phi_{2}\right),
$$

where

$$
\begin{aligned}
& \phi_{1}(\xi)= \begin{cases}\mathcal{R}_{\mathrm{LWR}}\left[\rho_{L}, \widetilde{\rho}\right](\xi) & \text { if } \xi<v_{R}, \\
\rho_{R} & \text { if } \xi \geqslant v_{R},\end{cases} \\
& \phi_{2}(\xi)= \begin{cases}w_{L} & \text { if } \xi<v_{R}, \\
w_{R} & \text { if } \xi \geqslant v_{R},\end{cases}
\end{aligned}
$$

$\tilde{\rho}=p^{-1}\left(w_{L}-\min \left\{w_{L}, v_{R}\right\}\right)$ and $\mathcal{R}_{\mathrm{LWR}}$ is the Riemann solver associated to LWR model (9) with $v(\rho)=w_{L}-$ $p(\rho)$, see Definition 4 .

Proposition 15. For any $W_{L}=\left(\rho_{L}, w_{L}\right), W_{R}=$ $\left(\rho_{R}, w_{R}\right) \in \Omega$, we have that

$$
W(t, x)=\mathcal{R}_{\mathrm{ARZ}}\left[W_{L}, W_{R}\right](x / t)
$$

is a self-similar weak solution of Riemann problem (30).

Proof. The initial condition in (30) is clearly satisfied. Hence we are left to show that $W$ satisfies ARZ model (29), at least in a weak sense.

The case $w_{L}=w_{R}$ is straightforward because then the $w$-component of $W$ is constant, $w \equiv w_{L, R}$, and $W$ satisfies (29) because its $\rho$-component satisfies (9) with $v(\rho)=w_{L, R}-p(\rho)$.

Assume $w_{L} \neq w_{R}$. The simplest way to obtain a weak formulation of (29) is to rewrite it as a $2 \times 2$ system of conservation laws. Let $y=\rho w$ be the generalized momentum of the vehicles. By introducing in ARZ model (29) the conservative variables $U=(\rho, y)$, we obtain the $2 \times 2$ system of conservation laws

$$
\partial_{t} U+\partial_{x} F(U)=\left(\begin{array}{l}
0 \\
0
\end{array}\right)
$$

where, with a slight abuse of notations, $F(U)=U v(U)$ with

$$
v(U)=\frac{y}{\rho}-p(\rho) .
$$

We stress that the above expression of the velocity in terms of the conservative variables $U$ is not well defined at the vacuum $\rho=0$; for this reason (29) and (31) are equivalent only away from the vacuum $\rho=0$. The weak formulation of (31) is

$$
\begin{aligned}
& \left\{\begin{array}{l}
\int_{\mathbb{R}} \int_{0}^{+\infty}\left(\rho \partial_{t} \varphi+v \rho \partial_{x} \varphi\right) \mathrm{d} t \mathrm{~d} x=0 \\
\int_{\mathbb{R}} \int_{0}^{+\infty} w\left(\rho \partial_{t} \varphi+v \rho \partial_{x} \varphi\right) \mathrm{d} t \mathrm{~d} x=0
\end{array}\right. \\
& \forall \varphi \in \mathbf{C}_{\mathbf{c}}^{\infty}((0,+\infty) \times \mathbb{R} ; \mathbb{R})
\end{aligned}
$$

Consider a test function $\varphi$ and let $D \subset \mathbb{R}^{2}$ be its compact support. We distinguish the following cases:

- If $D \subset\left\{(t, x) \in(0,+\infty) \times \mathbb{R}: x>v_{R} t\right\}$, then

$$
W \equiv\left(\begin{array}{c}
\rho_{R} \\
w_{R}
\end{array}\right) \quad \text { in } D .
$$

Hence $W$ is constant in $D$ and therefore satisfies (32).

- If $D \subset\left\{(t, x) \in(0,+\infty) \times \mathbb{R}: x<v_{R} t\right\}$, then

$$
W \equiv\left(\begin{array}{c}
\rho \\
w_{L}
\end{array}\right) \quad \text { in } D,
$$

where $\rho(t, x)=\mathcal{R}_{\mathrm{LWR}}\left[\rho_{L}, \rho_{R}\right](x / t)$ is a weak solution of the Riemann problem for LWR model (10) with $f(\rho)=\rho\left(w_{L}-p(\rho)\right)$. Therefore also in this case (32) easily follows.

- Assume that

$$
\begin{aligned}
& D_{-}=D \cap\left\{(x, t) \in \mathbb{R} \times(0,+\infty): x<v_{R} t\right\} \neq \emptyset, \\
& D_{+}=D \cap\left\{(x, t) \in \mathbb{R} \times(0,+\infty): x>v_{R} t\right\} \neq \emptyset .
\end{aligned}
$$

By construction $W$ is piecewise $\mathbf{C}^{\mathbf{1}}$ and, beside $x=$ $v_{R} t$, there exist at most other two straight lines across which $W$ is not $\mathbf{C}^{\mathbf{1}}$. For this reason it is not restrictive to assume that $D$ is sufficiently small so 


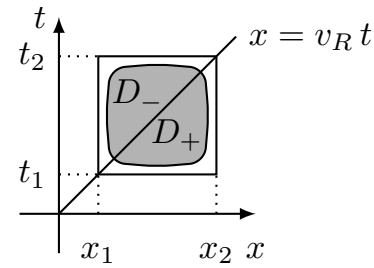

Fig. 19.: Notations used in the proof of Proposition 15.

that $W$ is $\mathbf{C}^{\mathbf{1}}$ both in $D_{-}$and $D_{+}$. Fix $x_{1}, x_{2} \in \mathbb{R}$ and $t_{1}, t_{2}>0$, see Fig. 19, such that

$$
D \subseteq\left[x_{1}, x_{2}\right] \times\left[t_{1}, t_{2}\right] .
$$

We have then

$\iint_{D_{-}}\left(\rho \partial_{t} \varphi+v(\rho, w) \rho \partial_{x} \varphi\right) \mathrm{d} x \mathrm{~d} t$

$=\int_{t_{1}}^{t_{2}} \int_{x_{1}}^{v_{R} t}\left(\rho \partial_{t} \varphi+v\left(\rho, w_{L}\right) \rho \partial_{x} \varphi\right) \mathrm{d} x \mathrm{~d} t$

$=\int_{t_{1}}^{t_{2}}\left(\frac{\mathrm{d}}{\mathrm{d} t}\left(\int_{x_{1}}^{v_{R} t} \rho \varphi \mathrm{d} x\right)-v_{R}[\rho \varphi]_{x=v_{R}}-\int_{x_{1}}^{v_{R} t} \partial_{t} \rho \varphi \mathrm{d} x\right) \mathrm{d} t$

$+\int_{t_{1}}^{t_{2}} \int_{x_{1}}^{v_{R} t}\left(\partial_{x}\left(v\left(\rho, w_{L}\right) \rho \varphi\right)-\partial_{x}\left(v\left(\rho, w_{L}\right) \rho\right) \varphi\right) \mathrm{d} x \mathrm{~d} t$

$=-\int_{t_{1}}^{t_{2}} v_{R} \tilde{\rho} \varphi\left(t, v_{R} t\right) \mathrm{d} t-\int_{t_{1}}^{t_{2}} \int_{x_{1}}^{v_{R} t} \partial_{t} \rho \varphi \mathrm{d} x \mathrm{~d} t$

$+\int_{t_{1}}^{t_{2}} v\left(\widetilde{\rho}, w_{L}\right) \widetilde{\rho} \varphi\left(t, v_{R} t\right) \mathrm{d} t-\int_{t_{1}}^{t_{2}} \int_{x_{1}}^{v_{R} t} \partial_{x}\left(v\left(\rho, w_{L}\right) \rho\right) \varphi \mathrm{d} x \mathrm{~d} t$

$=\int_{t_{1}}^{t_{2}}\left(v\left(\widetilde{\rho}, w_{L}\right)-v_{R}\right) \widetilde{\rho} \varphi\left(t, v_{R} t\right) \mathrm{d} t$

$-\int_{t_{1}}^{t_{2}} \int_{x_{1}}^{v_{R} t}\left(\partial_{t} \rho+\partial_{x}\left(v\left(\rho, w_{L}\right) \rho\right)\right) \varphi \mathrm{d} x \mathrm{~d} t=0$

because $\rho$ is a classical $\mathbf{C}^{\mathbf{1}}$-solution of (9) with $v(\rho)=$ $v\left(\rho, w_{L}\right)$ in $D_{-}$, and because either $v\left(\widetilde{\rho}, w_{L}\right)=v_{R}$, or $v\left(\widetilde{\rho}, w_{L}\right)=w_{L}$ and $\widetilde{\rho}=0$. Furthermore we have that

$\iint_{D_{+}}\left(\rho \partial_{t} \varphi+v(\rho, w) \rho \partial_{x} \varphi\right) \mathrm{d} x \mathrm{~d} t$

$=\int_{t_{1}}^{t_{2}} \int_{v_{R} t}^{x_{2}}\left(\rho_{R} \partial_{t} \varphi+v\left(W_{R}\right) \rho_{R} \partial_{x} \varphi\right) \mathrm{d} x \mathrm{~d} t$

$=\int_{t_{1}}^{t_{2}}\left(\frac{\mathrm{d}}{\mathrm{d} t}\left(\int_{v_{R} t}^{x_{2}} \rho_{R} \varphi \mathrm{d} x\right)+v_{R} \rho_{R} \varphi\left(t, v_{R} t\right)-v\left(W_{R}\right) \rho_{R} \varphi\left(t, v_{R} t\right)\right) \mathrm{d} t$

$=\left(v_{R}-v\left(W_{R}\right)\right) \rho_{R} \int_{t_{1}}^{t_{2}} \varphi\left(t, v_{R} t\right) \mathrm{d} t=0$

because by definition $v\left(W_{R}\right)=v_{R}$. At last, from the above computations easily follow that

$$
\begin{aligned}
& \iint_{D_{-}} w\left(\rho \partial_{t} \varphi+v(\rho, w) \rho \partial_{x} \varphi\right) \mathrm{d} x \mathrm{~d} t \\
& =w_{L} \iint_{D_{-}}\left(\rho \partial_{t} \varphi+v\left(\rho, w_{L}\right) \rho \partial_{x} \varphi\right) \mathrm{d} x \mathrm{~d} t=0
\end{aligned}
$$

$$
\begin{aligned}
& \iint_{D_{+}} w\left(\rho \partial_{t} \varphi+v(\rho, w) \rho \partial_{x} \varphi\right) \mathrm{d} x \mathrm{~d} t \\
& =w_{R} \iint_{D_{+}}\left(\rho \partial_{t} \varphi+v\left(\rho, w_{R}\right) \rho \partial_{x} \varphi\right) \mathrm{d} x \mathrm{~d} t=0 .
\end{aligned}
$$

This concludes the proof.

Notice that if $w_{L}=w_{R}$ then $\widetilde{\rho}=\rho_{R}$ and the weak solution $W$ has a rarefaction if $\rho_{L} \geqslant \rho_{R}$, or a shock if $\rho_{L}<\rho_{R}$. On the other hand, if $w_{L} \neq w_{R}$, then the weak solution $W$ has a discontinuity across $x=v_{R} t$, which is called contact discontinuity. We recall that an acceleration corresponds to a rarefaction wave and braking produces a shock wave. About the contact discontinuities, they simply separate vehicles with different Lagrangian markers. We clarify this point with the following example.

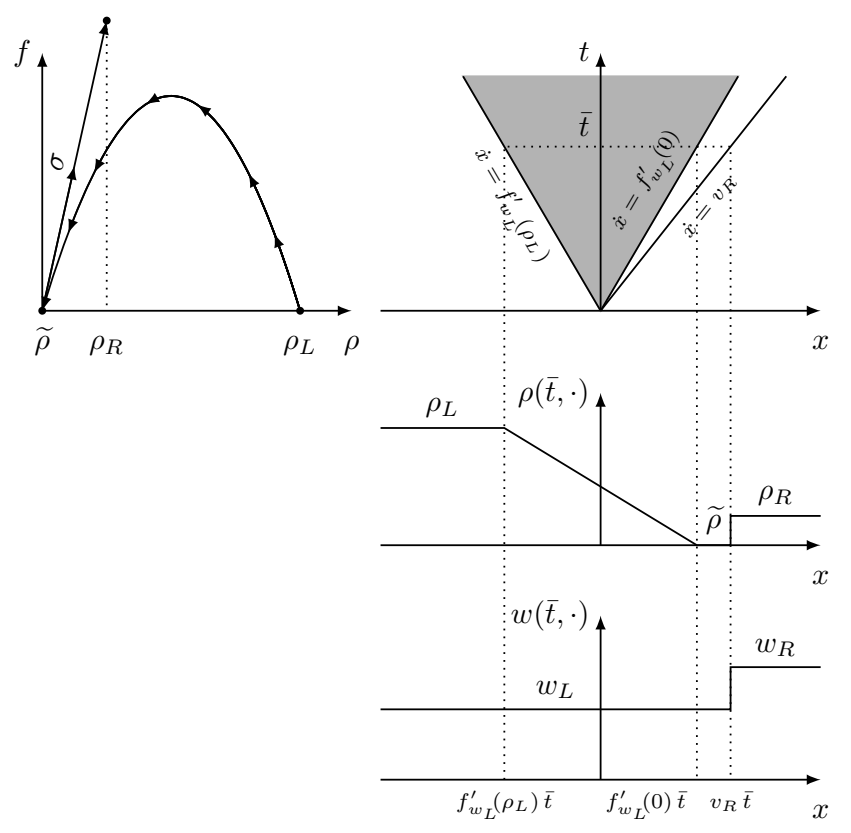

Fig. 20.: A rarefaction wave followed by a contact discontinuity. On the left we represent the fundamental diagram. On the right we give the solution in the $(x, t)$-plane, above, and the two components of its profiles $x \mapsto \rho(\bar{t}, x)$ and $x \mapsto w(\bar{t}, x)$ computed at a time $\bar{t}>0$, below.

Example 16 (Rarefaction wave+contact discontinuity). Consider "slow-long" and "fast-short" vehicles that at 
time $t=0$ are respectively in $(-\infty, 0)$ and $[0,+\infty)$. More precisely, assume that the vehicles in $x<0$ have Lagrangian marker $w_{L}$ and are "bumper-to-bumper", whereas those in $x \geqslant 0$ have Lagrangian marker $w_{R}$ and velocity $v_{R}$, with $0<w_{L}<v_{R}<w_{R}$. The corresponding model is given by Riemann problem (30) with

$$
\rho_{L}=p^{-1}\left(w_{L}\right), \quad \rho_{R}=p^{-1}\left(w_{R}-v_{R}\right) .
$$

In this case $\widetilde{\rho}=p^{-1}\left(w_{L}-\min \left\{w_{L}, v_{R}\right\}\right)=p^{-1}(0)=0$. The solution, see Fig. 20, has a rarefaction followed by contact discontinuity, has $\rho$-component

$$
\rho(t, x)= \begin{cases}\rho_{L} & \text { if } x<a\left(\rho_{L}\right) t \\ \left(f_{w_{L}}^{\prime}\right)^{-1}(x / t) & \text { if } f_{w_{L}}^{\prime}\left(\rho_{L}\right) t \leqslant x<f_{w_{L}}^{\prime}(0) t, \\ 0 & \text { if } f_{w_{L}}^{\prime}(0) t \leqslant x<v_{R} t \\ \rho_{R} & \text { if } x \geqslant v_{R} t\end{cases}
$$

where $f_{w_{L}}(\rho)=\rho v\left(\rho, w_{L}\right)=\rho\left(w_{L}-p(\rho)\right)$, and $w$ component

$$
w(t, x)= \begin{cases}w_{L} & \text { if } x<v_{R} t \\ w_{R} & \text { if } x \geqslant v_{R} t\end{cases}
$$

Remark 17. Some comments on the above example are in order. Even if the vacuum is not initially present, it arises immediately after $t=0$. This has a physical motivation: the slow vehicle cannot reach the speed $v_{R}$ of the fast vehicle because $w_{L}<v_{R}$, for this reason a gap is created between the two families of vehicles. As a consequence, the total variation of the $\rho$-component of the solution increases instantaneously at time $t=0^{+}$. On the contrary, it is easy to see that the total variation of the solution does not increase in the Riemann invariant coordinates $(v, w)$. For this reason existence results for ARZ model are based on estimates obtained in the Riemann invariant coordinates. At last, it is worth to mention that, at least away from the vacuum, $2 \times 2$ system of conservation laws (31) is a Temple ${ }^{60}$ system: the Lax curves in the Riemann invariant coordinates are straight lines.

Remark 18. In Ref. [61] it is proved that no Kruzhkov type condition can select a unique solution for ARZ model. Roughly speaking, the Kruzhkov type conditions can prohibit "wrong shocks" but it cannot prohibit "wrong contact discontinuities" adjacent to vacuum states. As a consequence, if for instance $W_{L}, W_{R} \in \Omega$ have $w_{L}=w_{R}, \rho_{L} \neq 0$ and $\rho_{R}=0$, then both the weak solutions

$$
\begin{aligned}
& W_{1}(t, x)=\left(\mathcal{R}_{\mathrm{LWR}}\left[\rho_{L}, \rho_{R}\right](x / t), w_{L, R}\right), \\
& W_{2}(t, x)= \begin{cases}W_{L} & \text { if } x<v_{L} t, \\
W_{R} & \text { if } x \geqslant v_{L} t,\end{cases}
\end{aligned}
$$

satisfy any Kruzhkov type condition with the equality, even if only $W_{1}$ is physically reasonable.

A possible way to uniquely select a solution is by requiring that it can be obtained as a many-particle limit analogous to that described in Section 4 for LWR model, see Ref. [17].

\section{Bottlenecks}

In this section we generalize both models (6) and (9) to reproduce the effects of an exit door placed at $x=0$ on the evacuation of a narrow corridor, see Fig. 21.

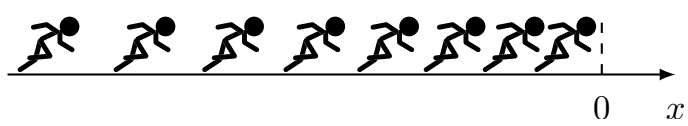

Fig. 21.: Evacuation of a corridor through an exit door.

The main feature of a door is given by its capacity $Q$, which is the maximum number of pedestrians that can flow through it in a given time interval. For this reason at the door position we impose the constraint on the flow

$$
f(\rho) \leqslant Q .
$$

It is reasonable to assume that

$$
Q \in\left(0, f_{\max }\right),
$$

where $f_{\max }=\max \left\{f(\rho): \rho \in\left[0, \rho_{\max }\right]\right\}$.

\subsection{Microscopic model}

At the microscopic level we have to rewrite condition (33) on the flow in terms of velocity function. This can be achieved by considering velocity function

$$
v_{\varepsilon}(x, \rho)= \begin{cases}\frac{Q}{f_{\max }} v(\rho) & \text { if }|x| \leqslant \varepsilon, \\ v(\rho) & \text { otherwise, }\end{cases}
$$

where $2 \varepsilon>0$ is the size of the door. Indeed (7) leads then to the flux function

$$
f_{\varepsilon}(x, \rho)=\rho v_{\varepsilon}(x, \rho),
$$


which attains values in $[0, Q]$ for any $x \in[-\varepsilon, \varepsilon]$. By introducing such velocity function in (6) we obtain the FTL model

$$
\begin{cases}\dot{x}_{1}(t)=v_{\max }, & \\ \dot{x}_{i}(t)=v_{\varepsilon}\left(x_{i}(t), \frac{1}{x_{i-1}(t)-x_{i}(t)}\right), & i \in\{2, \ldots, n\}, \\ x_{i}(0)=\bar{x}_{i}, & i \in\{1, \ldots, n\} .\end{cases}
$$

\subsection{Macroscopic model}

Differently from the microscopic level, at the macroscopic level it is not reasonable to consider the size of the door. For this reason condition (33) has to be imposed at $x=0$. As a result, the corresponding macroscopic model is given by LWR model (9) coupled with the point constraint on the flow

$$
f(t, 0) \leqslant Q .
$$

Notice that the classical solution selected by Kruzhkov condition (22) does not in general satisfy (35), as the following example shows.

Example 19. Fix $\rho_{0} \in\left(0, \rho_{\max }\right)$ and consider the Cauchy problem for LWR model (9) with constant initial condition

$$
\rho(0, x)=\rho_{0} .
$$

Its unique weak solution satisfying Kruzhkov condition (22) is the constant function $\rho \equiv \rho_{0}$. However, if $f\left(\rho_{0}\right)>$ $Q$ then such solution does not satisfy (35).

For this reason we introduce a new selection criterion of weak solution, and a new Riemann solver $\mathcal{R}_{Q}$, which we call constrained Riemann solver. To do so we can adapt the limiting process described in Section 4 as follows. On the basis of microscopic model (34), we consider

$$
\begin{cases}\dot{x}_{0}(t)=v_{\max }, & \\ \dot{x}_{i}(t)=v_{\varepsilon}\left(x_{i}(t), \frac{\ell}{x_{i-1}(t)-x_{i}(t)}\right), & i \in\{1, \ldots, n\}, \\ x_{i}(0)=\bar{x}_{i}, & i \in\{0, \ldots, n\},\end{cases}
$$

where $x_{0}, \ldots, x_{n} \in \mathbb{R}$ represent the positions of $n+1$ pedestrians, $\bar{x}_{n}<\ldots<\bar{x}_{0}$ are their initial positions, and $2 \varepsilon>0$ is the size of the door. Introduce then the corresponding discrete density

$$
\rho_{\varepsilon}^{n}(t, x)=\sum_{i=1}^{n} \frac{\ell}{x_{i}(t)-x_{i-1}(t)} \mathbb{1}_{\left[x_{i-1}(t), x_{i}(t)\right)}(x) .
$$

By letting $n$ go to infinity and $\varepsilon$ go to zero, we obtain a a weak solution satisfying both (9) and (35), and we call it constrained solution.

The corresponding constrained Riemann solver $\mathcal{R}_{Q}$ is described below. Let $\check{\rho}_{Q}, \hat{\rho}_{Q} \in\left[0, \rho_{\max }\right]$ be implicitly defined by

$$
f\left(\hat{\rho}_{Q}\right)=Q=f\left(\check{\rho}_{Q}\right), \quad 0 \leqslant \check{\rho}_{Q} \leqslant \rho_{\mathrm{m}} \leqslant \hat{\rho}_{Q},
$$

where $\rho_{\mathrm{m}} \in\left(0, \rho_{\max }\right)$ is the maximum of $f$, namely $f\left(\rho_{\mathrm{m}}\right)=f_{\max }$. Then $\mathcal{R}_{Q}:\left[0, \rho_{\max }\right]^{2} \rightarrow \mathbf{B V}\left(\mathbb{R} ;\left[0, \rho_{\max }\right]\right)$ is given by

$$
\mathcal{R}_{Q}\left[\rho_{L}, \rho_{R}\right](\xi) \doteq \begin{cases}\mathcal{R}_{\mathrm{LWR}}\left[\rho_{L}, \hat{\rho}_{Q}\right](\xi) & \text { if } \xi<0, \\ \mathcal{R}_{\mathrm{LWR}}\left[\check{\rho}_{Q}, \rho_{R}\right](\xi) & \text { if } \xi \geqslant 0 .\end{cases}
$$

A case by case study shows that $\mathcal{R}_{Q}\left[\rho_{L}, \rho_{R}\right]$ does not coincide with $\mathcal{R}_{\mathrm{LWR}}\left[\rho_{L}, \rho_{R}\right]$ if and only if $f\left(\mathcal{R}_{\mathrm{LWR}}\left[\rho_{L}, \rho_{R}\right]\right)(0)>Q$.

The constrained solution can also be obtained by applying a WFT algorithm analogous to that described in Section 3.2, but with $\mathcal{R}_{Q}$ replacing $\mathcal{R}_{\text {LWR }}$ any time an interaction occurs at the constraint position $x=0$, see Ref. [62] for the proof.

It is possible to show that the constrained solution is actually the unique weak solution of (9) satisfying the pointwise constraint condition (35) for a.e. $t>0$, together with the Kruzhkov type condition

$$
\begin{array}{r}
\int_{\mathbb{R}} \int_{0}^{+\infty}\left[|\rho(t, x)-k| \partial_{t} \varphi(t, x)+F(\rho(t, x), k) \partial_{x} \varphi(t, x)\right] \mathrm{d} t \mathrm{~d} x \\
+2 \int_{\mathbb{R}_{+}}[f(k)-\min \{f(k), Q\}] f(k) \varphi(t, 0) \mathrm{d} t \geqslant 0 \\
\forall \varphi \in \mathbf{C}_{\mathbf{c}}^{\infty}((0,+\infty) \times \mathbb{R} ;[0,+\infty)), k \in\left[0, \rho_{\max }\right],
\end{array}
$$

where $F$ is given by (23).

In the next example we apply $\mathcal{R}_{Q}$ to construct the constrained solution of the problem considered in Example 19.

Example 20. Fix $\rho_{0} \in\left(0, \rho_{\max }\right)$ such that $f\left(\rho_{0}\right)>Q$. Consider again the Cauchy problem for LWR model (9) with initial condition (36). By applying the constrained Riemann solver $\mathcal{R}_{Q}$ we obtain the constrained solution

$$
\rho(t, x)=\mathcal{R}_{Q}\left[\rho_{0}, \rho_{0}\right](x / t),
$$

which satisfies constraint condition (35), see Fig. 22.

Remark 21. Some comments on the above example are in order. The constrained solution does not satisfy the 


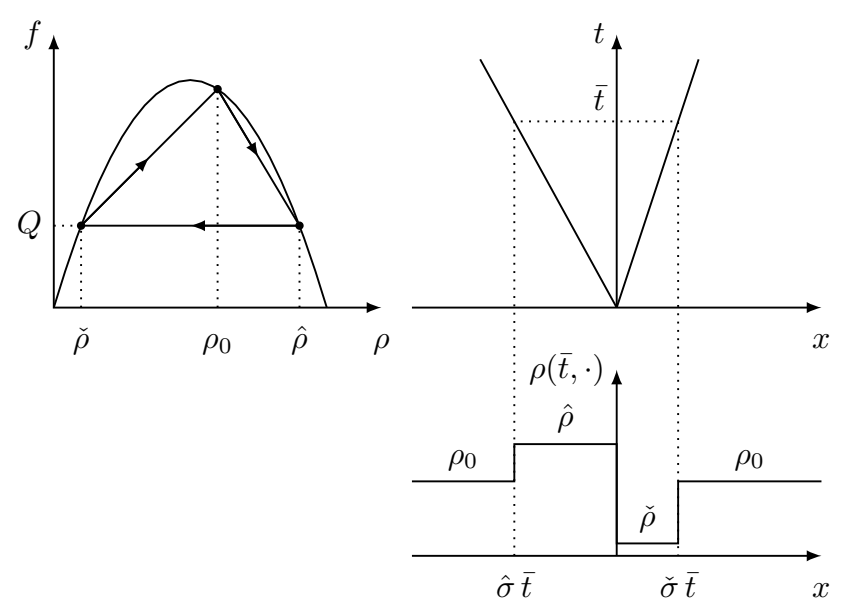

Fig. 22.: The constrained solution of the LWR model with point constraint (9), (35). Above $\hat{\rho}=\hat{\rho}_{Q}\left(\rho_{0}, \rho_{0}\right), \check{\rho}=\check{\rho}_{Q}\left(\rho_{0}, \rho_{0}\right), \hat{\sigma}=\sigma\left(\rho_{0}, \hat{\rho}_{Q}\right)$ and $\check{\sigma}=\sigma\left(\check{\rho}_{Q}, \rho_{0}\right)$.

maximum principle. Moreover, its total variation instantaneously increases at time $t=0^{+}$. We point out that the authors of Ref. [62] proved that the total variation of a constrained solution does not increase in the coordinate $\psi(\rho)=\int_{0}^{\rho}\left|f^{\prime}(r)\right| \mathrm{d} r$.

In the next two examples we show how Braess' paradox and faster-is-slower effects can be reproduced by the constrained LWR model (9), (35) in the framework of pedestrian flows.

Example 22. Fix $a<b<0$ and $0<q<Q<f_{\max }$. Consider a narrow corridor with an exit door placed at $x=0$. Assume that the capacity of the door is $q \in$ $\left(0, f_{\max }\right)$. Consider a crowd to be evacuated, which at time $t=0$ is uniformly distributed in $[a, b]$ with density $\rho_{\max }$. The resulting model is the Cauchy problem for LWR model (9) with point constraint on the flow (35) and initial condition

$$
\rho(0, x)=\rho_{\max } \mathbb{1}_{[a, b]}(x),
$$

where $\mathbb{1}_{[a, b]}$ is the indicator function of the interval $[a, b] \subset$ $\mathbb{R}$. The approximate solution can be constructed by applying a WFT analogous to that described in Section 3.2. Below we show some details of this construction for the most representative case, see Fig. 23.

- Fix $n \in \mathbb{N}$ sufficiently big. Introduce the mesh

$$
\mathcal{M}_{n}=\left\{j \frac{\check{\rho}_{q}}{n}: j \in[0, n] \cap \mathbb{Z}\right\}
$$
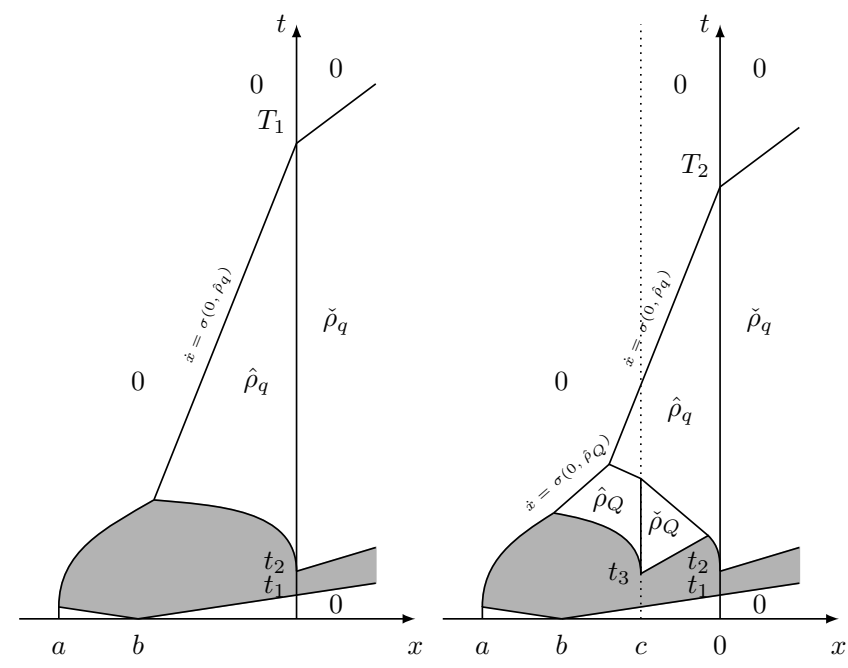

Fig. 23.: Left: Evacuation of a narrow corridor $(-\infty, 0)$ with an exit door placed at $x=0$ and a crowd initially uniformly distributed in $[a, b]$ with density $\rho=\rho_{\max }$. At time $t=t_{1}$ the first pedestrian crosses the door. At time $t=t_{2}$ the capacity $q$ of the exit door is reached and a queue appears in the upstream of the exit door. At time $t=T_{1}$ the corridor is evacuated. Right: Evacuation of a narrow corridor $(-\infty, 0)$ with two doors placed at $x=0$ and $x=c$, and a crowd initially uniformly distributed in $[a, b]$ with density $\rho=\rho_{\max }$. At time $t=t_{1}$ the first pedestrian crosses the door. At time $t=t_{2}$ the capacity $q$ of the exit door is reached and a queue appears in the upstream of the exit door. At time $t=t_{3}$ the capacity $Q$ of the door is reached and a queue appears in the upstream of the door at $x=c$. At time $t=T_{2}$ the corridor is evacuated. Notice that the evacuation time is higher in the case considered on the left, $T_{1}>T_{2}$.

$$
\begin{aligned}
& \cup\left\{\check{\rho}_{q}+j \frac{\hat{\rho}_{q}-\check{\rho}_{q}}{n}: j \in(0, n) \cap \mathbb{N}\right\} \\
& \cup\left\{\hat{\rho}_{q}+j \frac{\rho_{\max }-\hat{\rho}_{q}}{n}: j \in[0, n] \cap \mathbb{Z}\right\} .
\end{aligned}
$$

Let $\rho_{j} \in\left[0, \rho_{\max }\right]$ be such that $\mathcal{M}_{n}=\left\{\rho_{j}: j \in\right.$ $[0,3 n] \cap \mathbb{Z}\}$ and $\rho_{j}<\rho_{j+1}$. Observe that

$$
\rho_{0}=0, \quad \rho_{n}=\check{\rho}_{q}, \quad \rho_{2 n}=\hat{\rho}_{q}, \quad \rho_{3 n}=\rho_{\max } .
$$

- Let $\mathcal{R}_{\mathrm{LWR}}^{n}$ and $\mathcal{R}_{q}^{n}$ be the approximate Riemann solvers obtained from $\mathcal{R}_{\mathrm{LWR}}$ and $\mathcal{R}_{q}$ by discretizing the rarefactions along the mesh $\mathcal{M}_{n}$. We apply the 
approximate Riemann solvers at the discontinuities of the initial datum:

$$
x=0: \quad \mathcal{R}_{q}^{n}[0,0](\xi)=0,
$$$$
x=a: \mathcal{R}_{\mathrm{LWR}}^{n}\left[0, \rho_{\max }\right](\xi)= \begin{cases}0 & \text { if } \xi<0, \\ \rho_{\max } & \text { if } \xi \geqslant 0,\end{cases}
$$$$
x=b: \mathcal{R}_{\mathrm{LWR}}^{n}\left[\rho_{\max }, 0\right](\xi)=\left\{\begin{array}{rr}
\rho_{\max } & \text { if } \xi<0, \\
\rho_{j} & \text { if } \sigma\left(\rho_{j+1}, \rho_{j}\right) \leqslant \xi<\sigma\left(\rho_{j}, \rho_{j-1}\right), \\
& j \in[1,3 n-1] \cap \mathbb{N}, \\
0 & \text { if } \xi \geqslant \sigma\left(\rho_{1}, 0\right) .
\end{array}\right.
$$

- By shifting the solutions to the corresponding $x$ position and juxtaposing them, we have that for $t>0$ sufficiently small the approximate solution is

$$
\rho^{n}(t, x)= \begin{cases}0 & \text { if } x<a, \\ \rho_{\max } & \text { if } a \leqslant x<b+\sigma\left(\rho_{3 n}, \rho_{3 n-1}\right) t, \\ \rho_{j} & \text { if } \sigma\left(\rho_{j+1}, \rho_{j}\right) \leqslant \frac{x-b}{t}<\sigma\left(\rho_{j}, \rho_{j-1}\right), \\ & \quad j \in[1,3 n-1] \cap \mathbb{N}, \\ 0 & \text { if } x \geqslant b+\sigma\left(\rho_{1}, 0\right) t .\end{cases}
$$

- We apply $\mathcal{R}_{q}^{n}$ at $x=0$ each time a wave started from $x=b$ reaches $x=0$. Notice that the first wave reaches $x=0$ at time $t_{1}^{n}=-b / \sigma\left(\rho_{1}, \rho_{0}\right)$. The waves $\left(\rho_{j}, \rho_{j-1}\right)$ with $j \leqslant n$ cross $x=0$ unchanged because $f\left(\rho_{j-1}\right)<f\left(\rho_{j}\right) \leqslant q$ and therefore $\mathcal{R}_{q}^{n}\left[\rho_{j}, \rho_{j-1}\right] \equiv \mathcal{R}_{\mathrm{LWR}}^{n}\left[\rho_{j}, \rho_{j-1}\right]$. On the other hand, at time $t_{2}^{n}=-b / \sigma\left(\rho_{n+1}, \rho_{n}\right)$ the wave $\left(\rho_{n+1}, \rho_{n}\right)$ reaches $x=0$ and new waves are created because $f\left(\rho_{n+1}\right)>q$. More precisely, a shock $\left(\rho_{n+1}, \rho_{2 n}\right)$ and a stationary non-classical shock $\left(\rho_{2 n}, \rho_{n}\right)$ start from $x=0$ at time $t=t_{2}^{n}$ because

$$
\mathcal{R}_{q}^{n}\left[\rho_{n+1}, \rho_{n}\right](\xi)= \begin{cases}\rho_{n+1} & \text { if } \xi<\sigma\left(\rho_{n+1}, \rho_{2 n}\right), \\ \rho_{2 n} & \text { if } \sigma\left(\rho_{n+1}, \rho_{2 n}\right) \leqslant \xi<0, \\ \rho_{n} & \text { if } \xi \geqslant 0 .\end{cases}
$$

The new shock interacts then with another wave started from $x=b$ and a new shock is created because

$$
\mathcal{R}_{\mathrm{LWR}}^{n}\left[\rho_{n+2}, \rho_{2 n}\right](\xi)= \begin{cases}\rho_{n+2} & \text { if } \xi<\sigma\left(\rho_{n+2}, \rho_{2 n}\right), \\ \rho_{2 n} & \text { if } \xi \geqslant \sigma\left(\rho_{n+2}, \rho_{2 n}\right) .\end{cases}
$$

The new shock interacts then with another wave started from $x=b$ and a new shock is created, and so on.

- The stationary shock started from $x=a$ interacts with the leftmost wave started from $x=b$. The result of this interaction is a shock because

$$
\mathcal{R}_{\mathrm{LWR}}^{n}\left[\rho_{0}, \rho_{3 n-1}\right](\xi)= \begin{cases}\rho_{0} & \text { if } \xi<\sigma\left(\rho_{0}, \rho_{3 n-1}\right), \\ \rho_{3 n-1} & \text { if } \xi \geqslant \sigma\left(\rho_{0}, \rho_{3 n-1}\right) .\end{cases}
$$

The new shock interacts then with another wave started from $x=b$ and a new shock is created because

$$
\mathcal{R}_{\text {LWR }}^{n}\left[\rho_{0}, \rho_{3 n-2}\right](\xi)= \begin{cases}\rho_{0} & \text { if } \xi<\sigma\left(\rho_{0}, \rho_{3 n-2}\right), \\ \rho_{3 n-2} & \text { if } \xi \geqslant \sigma\left(\rho_{0}, \rho_{3 n-2}\right) .\end{cases}
$$

The new shock interacts then with another wave started from $x=b$ and a new shock is created, and so on.

- After some time, the interaction between the waves $\left(\rho_{0}, \rho_{j}\right)$ and $\left(\rho_{j}, \rho_{2 n}\right)$ occur in $(-\infty, 0)$. The result is the shock $\left(\rho_{0}, \rho_{2 n}\right)$ because

$$
\mathcal{R}_{\mathrm{LWR}}^{n}\left[\rho_{0}, \rho_{2 n}\right](\xi)= \begin{cases}\rho_{0} & \text { if } \xi<\sigma\left(\rho_{0}, \rho_{2 n}\right), \\ \rho_{2 n} & \text { if } \xi \geqslant \sigma\left(\rho_{0}, \rho_{2 n}\right) .\end{cases}
$$

- The shock $\left(\rho_{0}, \rho_{2 n}\right)$ eventually reaches $x=0$ at time $T^{n}$ and interacts with the stationary non-classical shock $\left(\rho_{2 n}, \rho_{n}\right)$. The result of the interaction is the shock $\left(\rho_{0}, \rho_{n}\right)$ because

$$
\mathcal{R}_{q}^{n}\left[\rho_{0}, \rho_{2 n}\right](\xi)= \begin{cases}\rho_{0} & \text { if } x<\sigma\left(\rho_{0}, \rho_{n}\right), \\ \rho_{n} & \text { if } x \geqslant \sigma\left(\rho_{0}, \rho_{n}\right) .\end{cases}
$$

By letting then $n$ got to infinity we obtain the solution represented in Fig. 23.

Consider now a further wider door placed at $x=c$ with $c \in(b, 0)$. Denote by $Q \in\left(q, f_{\max }\right)$ its capacity. The corresponding model is

$$
\left\{\begin{array}{l}
\partial_{t} \rho+\partial_{x} f(\rho)=0 \\
f(\rho)(t, c) \leqslant Q \\
f(\rho)(t, 0) \leqslant q
\end{array}\right.
$$

We can adapt the construction described above and obtain the solution represented in Fig. 23.

Notice that, at least in the cases under consideration, the evacuation is lower when two doors are present, $T_{2}<$ $T_{1}$. The reason is that $\sigma\left(0, \hat{\rho}_{Q}\right)>\sigma\left(0, \hat{\rho}_{q}\right)$. Therefore this basic model is able to reproduce the Braess' paradox.

Example 23. Consider the Cauchy problem for LWR model (9) with point constraint on the flow (35)

$$
\left\{\begin{array}{l}
\partial_{t} \rho+\partial_{x} f_{V}(\rho)=0 \\
f_{V}(\rho)(t, 0) \leqslant q \\
\rho(0, x)=\rho_{\max } \mathbb{1}_{[a, b]}(x)
\end{array}\right.
$$




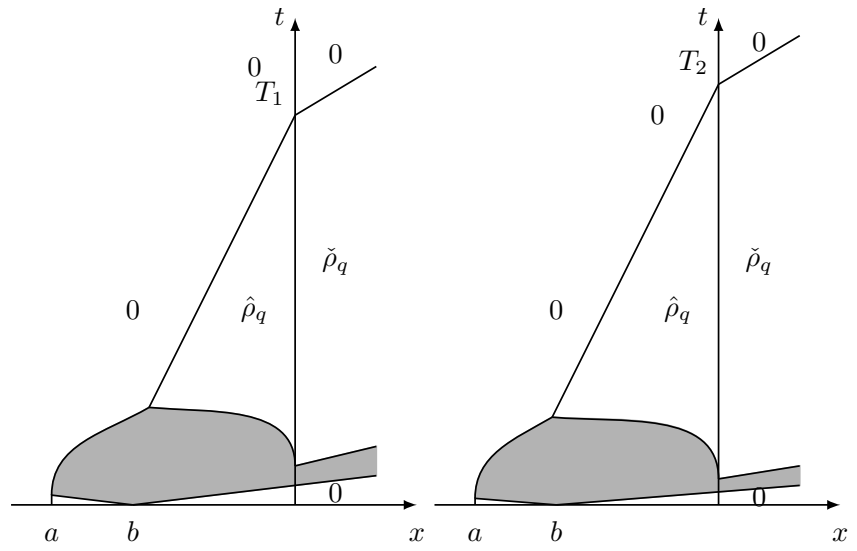

Fig. 24.: Evacuation of a narrow corridor $(-\infty, 0)$ with a door placed at $x=0$ and a crowd initially uniformly distributed in $[a, b]$ with density $\rho=\rho_{\max }$. The maximal speed is higher in the case considered on the right. Also the evacuation time is higher in the case considered on the right, $T_{2}>T_{1}$. where

$$
f_{V}(\rho)=V \rho\left(1-\frac{\rho}{\rho_{\max }}\right) .
$$

Notice that by definition the maximal velocity is $v_{\max }=$ $V$. The construction of the solution is analogous to that in Example 22. The evacuation time is clearly a function of the maximal velocity, $T=T(V)$. In Fig. 24 we compare two solutions corresponding to two different values of $V$ and show that, at least in the cases under consideration, the evacuation time $T(V)$ is higher for the higher values of $V$. This shows that this basic model is able to reproduce also the faster-is-slower effect.

In some real life applications, the constraint $Q$ is not constant. In the next examples we introduce more general expressions of the constraint $Q$ motivated by some applications and give some references for their study.

Example 24. If we replace the door with a traffic light, then $Q$ depends on time ${ }^{9,62}$ and takes the form

$$
Q(t)= \begin{cases}f_{\max } & \text { if the traffic light is green, } \\ 0 & \text { if the traffic light is red. }\end{cases}
$$

Example 25. Despite the common belief, the capacity of a door is not constant. In fact it can drop when highdensity conditions occur at the door position. For this reason in Ref. [8] the authors introduce a threshold value $\rho_{\mathrm{c}} \in\left(0, \rho_{\mathrm{max}}\right)$ to distinguish low and high densities, and assume that the capacity $Q$ takes the form

$$
Q(\rho)= \begin{cases}q_{\mathrm{l}} & \text { if } \rho<\rho_{\mathrm{c}}, \\ q_{\mathrm{h}} & \text { if } \rho \geqslant \rho_{\mathrm{c}}\end{cases}
$$

with $0<q_{\mathrm{h}}<q_{1}<f_{\max }$.

We recall that in Ref. [10] it is assumed a more regular decay of the capacity. For this reason it is introduced a non-local constraint depending on a weighted average density of pedestrians in the upstream of the door with the form

$$
Q(\rho, t)=q\left(\int_{\mathbb{R}_{-}} w(x) \rho(t, x) \mathrm{d} x\right),
$$

where $w:(-\infty, 0] \rightarrow[0,+\infty)$ is the compactly supported non-decreasing weight function used for the average density and $q:[0,+\infty) \rightarrow(0,+\infty)$ is non-increasing and prescribes the maximal flow allowed through the door.

Example 26. Consider vehicular traffic through a toll booth, where the number of open gates is decided according to on line data.

- If the data are collected by a video camera, then we can take

$$
Q(\rho, t)=q\left(\int_{\mathbb{R}_{-}} \int_{0}^{t} w(x) \kappa(t-s) \rho(s, x) \mathrm{d} s \mathrm{~d} x\right),
$$

where $\operatorname{supp}(w)$ represents the area registered by the video camera and $\operatorname{supp}(\kappa)$ gives the period of time the data are taken into account.

- If the data come from a photo camera that shoots photos at times $t_{i}$ of the area given by $\operatorname{supp}(w)$, then we can take

$$
Q(\rho, t)=q\left(\sum_{t_{i}<t} \int_{\mathbb{R}_{-}} w(x) \kappa\left(t-t_{i}\right) \rho\left(t_{i}, x\right) \mathrm{d} x\right),
$$

where $\operatorname{supp}(\kappa)$ gives the period of time the data are taken into account.

- If the data come from local sensors located in $y_{i}$, then we can take

$Q(\rho, t)=q\left(\sum_{i=1}^{M}\left[+\int_{0}^{t} w^{\prime}\left(y_{i}\right) \bar{\kappa}(t-s) f(\rho)\left(s, y_{i}\right) \mathrm{d} s\right]\right)$,

where $\operatorname{supp}(\kappa)$ gives the period of time the data are taken into account. 
The above choices for $Q$ fall in the general framework of constraints

$$
Q: \mathbf{C}^{\mathbf{0}}\left([0, T] ; \mathbf{L}^{\mathbf{1}}(\mathbb{R} ; \mathbb{R})\right) \rightarrow \mathbf{L}^{\mathbf{1}}([0, T] ;[0, f(\bar{\rho})]),
$$

where the operator $Q$ may be non-local both in time and space, see Ref. [11]. Notice that a time delay in adapting the number of open gates to the available data can be easily modeled.

Further interesting developments of the theory of point constraint can be found in Ref. [63-69].

\section{A macroscopic model for pedestrian flows with panic}

In this section we develop a model for pedestrian flows that can reproduce panic behavior, see Ref. $[8,70,71]$. We
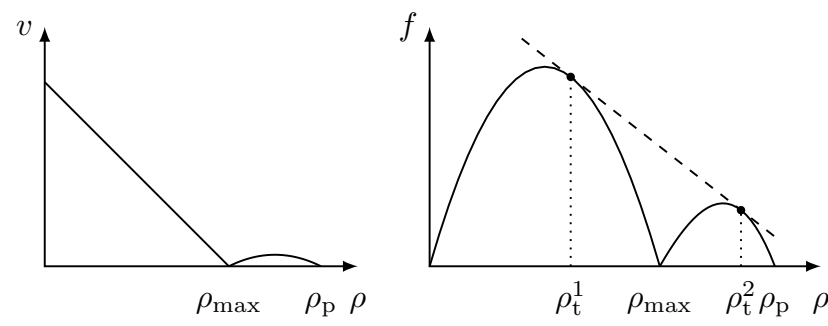

Fig. 25.: A velocity function and fundamental diagram accounting for panic densities. The dashed line is tangent to the fundamental diagram both at $\rho=\rho_{\mathrm{t}}^{1}$ and $\rho=\rho_{\mathrm{t}}^{2}$.

build it starting from the LWR model as follows:

- Beside the interval $\left[0, \rho_{\max }\right]$ of "standard" densities, we introduce the interval $\left(\rho_{\max }, \rho_{\mathrm{p}}\right]$ of densities corresponding to panic.

- We extend the fundamental diagram and the speed law to the whole density interval $\left[0, \rho_{\mathrm{p}}\right]$. As for LWR model, the velocity is assumed to be decreasing in the standard interval $\left(\rho_{\max }, \rho_{\mathrm{p}}\right]$. On the contrary, we assume that entering in the interval of densities $\left(\rho_{\max }, \rho_{\mathrm{p}}\right]$ the panic forces the velocity to increase. Since clearly $v\left(\rho_{\mathrm{p}}\right)=0$, we assume that $v$ is strictly concave in $\left(\rho_{\max }, \rho_{\mathrm{p}}\right]$. For simplicity in the construction given below, we assume that also $v\left(\rho_{\max }\right)=0$, see Fig. 25.

- The weak solution satisfying Kruzhkov condition (22) satisfies also the maximum principle, which implies that if the initial density takes values in the standard interval of densities, then panic will never arise. This is in contrast with what we know from empirical observations. For this reason we introduce a new Riemann solver that does not satisfy the maximum principle, so that the transition to panic can occur even if the initial density takes values in the standard interval.

According to the above last point, we have to introduce a new Riemann solver $\mathcal{R}_{\mathrm{p}}:\left[0, \rho_{\mathrm{p}}\right]^{2} \rightarrow \mathbf{B V}\left(\mathbb{R} ;\left[0, \rho_{\mathrm{p}}\right]\right)$. To do so we start by considering standard densities. Imagine we are (almost) the last in the queue. In this case there is no reason to feel in panic because we can avoid high densities by simply stopping. Furthermore, a sudden increase of the density may lead to panic. These two basic considerations lead us to introduce two thresholds: a "small" $r \in\left(0, \rho_{\max }\right)$ and a "big" $\Delta r \in\left(0, \rho_{\max }\right)$ that correspond to the two cases just described. These two thresholds select in the square of standard densities $\left[0, \rho_{\max }\right]^{2}$ a triangle

$$
\mathrm{T}=\left\{\left(\rho_{L}, \rho_{R}\right) \in\left[0, \rho_{\max }\right]^{2}: \rho_{L} \geqslant r, \rho_{R}-\rho_{L} \geqslant \Delta r\right\},
$$

such that if $\left(\rho_{L}, \rho_{R}\right) \in \mathrm{T}$, then $\mathcal{R}_{\mathrm{p}}\left[\rho_{L}, \rho_{R}\right]$ attains values also in the panic interval $\left(\rho_{\max }, \rho_{\mathrm{p}}\right]$. In the same spirit as in Ref. [72], for any $\left(\rho_{L}, \rho_{R}\right) \in \mathrm{T}$, let $\widetilde{\rho}_{L} \in\left(\rho_{\max }, \rho_{\mathrm{p}}\right]$ be implicitly selected by the conditions

$$
\widetilde{\rho}_{L} \in\left(\rho_{\max }, \rho_{\mathrm{p}}\right], \quad f^{\prime}\left(\widetilde{\rho}_{L}\right)=\sigma\left(\rho_{L}, \widetilde{\rho}_{L}\right),
$$

and define

$$
\mathcal{R}_{\mathrm{p}}\left[\rho_{L}, \rho_{R}\right](\xi)= \begin{cases}\rho_{L} & \text { if } \xi<\sigma\left(\rho_{L}, \widetilde{\rho}_{L}\right), \\ \mathcal{R}_{\mathrm{K}}\left[\widetilde{\rho}_{L}, \rho_{R}\right](\xi) & \text { if } \xi \geqslant \sigma\left(\rho_{L}, \widetilde{\rho}_{L}\right),\end{cases}
$$

where $\mathcal{R}_{\mathrm{K}}$ is the unique Riemann solver satisfying the Kruzhkov condition (22), see Fig. 26. Of course we need that

$$
\widetilde{r+\Delta r}<\widetilde{r}
$$

to avoid the overlapping of the two waves performed by (38).

Notice that $\mathcal{R}_{\mathrm{p}}$ is not $\mathbf{L}_{\text {loc }}^{\mathbf{1}}$-continuous in $\left[0, \rho_{\text {max }}\right]^{2}$. However we can extend $\mathcal{R}_{\mathrm{p}}$ to the whole of $\left[0, \rho_{\mathrm{p}}\right]^{2}$ in such a way to minimize its $\mathbf{L}_{\mathbf{l o c}}^{\mathbf{1}}$-discontinuity lines. Having this in mind, starting from $\left(\rho_{L}, \rho_{R}\right) \in \mathrm{T}$, we define

$$
\mathcal{R}_{\mathrm{p}}\left[\rho_{L}, \rho\right](\xi)= \begin{cases}\rho_{L} & \text { if } \xi<\sigma\left(\rho_{L}, \widetilde{\rho}\right), \\ \mathcal{R}_{\mathrm{K}}[\widetilde{\rho}, \rho](\xi) & \text { if } \xi \geqslant \sigma\left(\rho_{L}, \widetilde{\rho}\right),\end{cases}
$$




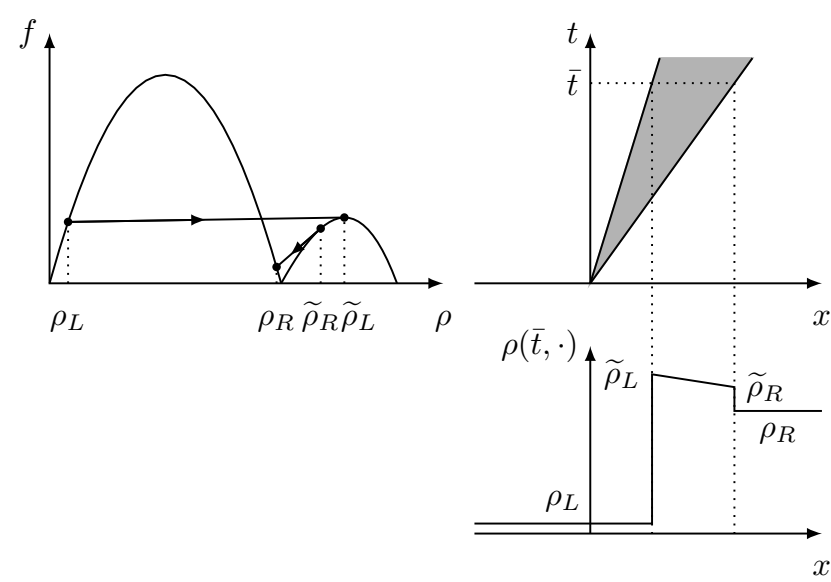

Fig. 26.: The solution $\rho(t, x)=\mathcal{R}_{\mathrm{p}}\left[\rho_{L}, \rho_{R}\right](x / t)$ in the case $\left(\rho_{L}, \rho_{R}\right) \in \mathrm{T}$. Notice that the maximum principle is violated and that panic densities arises even if the initial datum attains values in the standard interval of densities.

in analogy with (38) as long as this makes sense, namely for $\rho \in\left[\rho_{R}, \widetilde{\rho}\right]$. For $\rho \in\left(\widetilde{\rho}, \rho_{\mathrm{p}}\right]$, we keep the $\mathbf{L}_{\mathbf{l o c}^{-}}^{\mathbf{1}}$ continuity by defying

$$
\mathcal{R}_{\mathrm{p}}\left[\rho_{L}, \rho\right](\xi)= \begin{cases}\rho_{L} & \text { if } \xi<\sigma\left(\rho_{L}, \rho_{R}\right), \\ \rho_{R} & \text { if } \xi \geqslant \sigma\left(\rho_{L}, \rho_{R}\right) .\end{cases}
$$

Again, seeking to preserve $\mathbf{L}_{\text {loc }}^{\mathbf{1}}$-continuity, we are lead to define $\mathcal{R}_{\mathrm{p}}$ in $\left[0, \rho_{\max }\right] \times\left(\rho_{\max }, \rho_{\mathrm{p}}\right]$ by using $(38)$ if $\rho_{R} \leqslant \widetilde{\rho}_{L}$ and (39) otherwise. At last, in $\left(\rho_{\max }, \rho_{\mathrm{p}}\right] \times\left[0, \rho_{\mathrm{p}}\right]$ it si sufficient to simply take $\mathcal{R}_{\mathrm{p}}=\mathcal{R}_{\mathrm{K}}$.

On the basis of the above considerations, we introduce

$$
\mathrm{N}=\mathrm{T} \cup\left(\left[0, \rho_{\max }\right] \times\left[\rho_{\max }, \rho_{\mathrm{p}}\right]\right),
$$

where $\mathcal{R}_{\mathrm{p}}$ does not coincide with $\mathcal{R}_{\mathrm{K}}$, and give the following definition, see Fig. 27.

Definition 27. The Riemann solver $\mathcal{R}_{\mathrm{p}}:\left[0, \rho_{\mathrm{p}}\right]^{2} \rightarrow$ $\mathbf{B V}\left(\mathbb{R} ;\left[0, \rho_{\mathrm{p}}\right]\right)$ is defined as follows:

- If $\left(\rho_{L}, \rho_{R}\right) \in \mathbf{N}$, then we let

$$
\mathcal{R}_{\mathrm{p}}\left[\rho_{L}, \rho_{R}\right](\xi)= \begin{cases}\rho_{L} & \text { if } \xi<\sigma\left(\rho_{L}, \rho_{M}\right), \\ \mathcal{R}_{\mathrm{K}}\left[\rho_{M}, \rho_{R}\right](\xi) & \text { if } \xi \geqslant \sigma\left(\rho_{L}, \rho_{M}\right),\end{cases}
$$

where $\rho_{M}=\max \left\{\tilde{\rho}, \rho_{R}\right\}$, with $\widetilde{\rho} \in\left(\rho_{\max }, \rho_{\mathrm{p}}\right]$ implicitly selected by $(37)$.

- If $\left(\rho_{L}, \rho_{R}\right) \notin \mathrm{N}$, then we define $\mathcal{R}_{\mathrm{p}} \equiv \mathcal{R}_{\mathrm{K}}$.

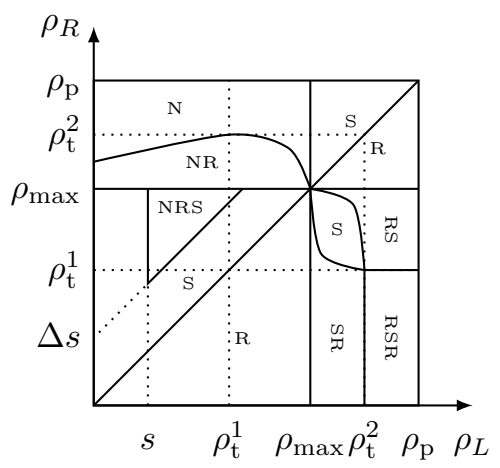

Fig. 27.: Representation of the Riemann solver $\mathcal{R}_{\mathrm{p}}$ given by Definition 27. Above $\rho_{\mathrm{t}}^{1}$ and $\rho_{\mathrm{t}}^{2}$ are the same as in Fig. 25. Moreover, for instance, NRS means that the solutions corresponding to data in that region have a non-classical shock, a rarefaction and a shock.

Further details on this model as well as the construction of a solution can be found in Ref. [73]. It is worth to underline here that the main assumptions of the model has been experimentally confirmed by studying the video of the crowd accident on the Jamarat bridge of 2006, see Ref. [74].

\section{Acknowledgments}

The author is member of GNAMPA. He acknowledges the support of the National Science Centre, Poland, Project "Mathematics of multi-scale approaches in life and social sciences" No. 2017/25/B/ST1/00051, of the INdAMGNAMPA Project 2019 "Equazioni alle derivate parziali di tipo iperbolico o non locale ed applicazioni" and of University of Ferrara, FIR Project 2019 "Leggi di conservazione di tipo iperbolico: teoria ed applicazioni".

\section{References}

1. R. L. Hughes, The flow of human crowds, Annual Review of Fluid Mechanics. 35(1), 169-182 (2003).

2. D. Helbing, I. Farkas, and T. Vicsek, Simulating dynamical features of escape panic, Nature. 407(6803), 487-490 (2000).

3. E. M. Cepolina, Phased evacuation: An optimisation model which takes into account the capacity drop phenomenon in pedestrian flows, Fire Safety Journal. 44(4), 532 - 544 (2009). ISSN 0379-7112.

4. S. Soria, R. Josens, and D. Parisi, Experimental evidence of the "faster is slower" effect in the evacuation of ants, 
February 25, 2020 16:29 Preprint-9×6 Book Title: Order, Disorder and Criticality: Advanced Problems of Preprint page 26 Phase Transition Theory. Ed. by Yu. Holovatch. Vol. 6, 2020, World Scientific, Singapore

Safety Science. 50(7), 1584 - 1588 (2012). ISSN 09257535 .

5. M. Twarogowska, P. Goatin, and R. Duvigneau, Macroscopic modeling and simulations of room evacuation, $A p$ plied Mathematical Modelling. 38(24), 5781 - 5795 (2014). ISSN 0307-904X.

6. R. M. Colombo and E. Rossi, Modelling crowd movements in domains with boundaries, IMA J. Appl. Math. 84(5), 833-853 (2019). ISSN 0272-4960.

7. E. Cristiani, F. S. Priuli, and A. Tosin, Modeling rationality to control self-organization of crowds: an environmental approach, SIAM J. Appl. Math. 75(2), 605-629 (2015). ISSN 0036-1399.

8. R. M. Colombo and M. D. Rosini, Pedestrian flows and non-classical shocks, Math. Methods Appl. Sci. 28(13), 1553-1567 (2005). ISSN 0170-4214.

9. R. M. Colombo, P. Goatin, and M. D. Rosini, On the modelling and management of traffic, ESAIM Math. Model. Numer. Anal. 45(5), 853-872 (2011). ISSN 0764$583 \mathrm{X}$.

10. B. Andreianov, C. Donadello, and M. D. Rosini, Crowd dynamics and conservation laws with nonlocal constraints and capacity drop, Math. Models Methods Appl. Sci. 24 (13), 2685-2722 (2014). ISSN 0218-2025.

11. B. Andreianov, C. Donadello, U. Razafison, and M. D. Rosini, Analysis and approximation of one-dimensional scalar conservation laws with general point constraints on the flux, J. Math. Pures Appl. (9). 116, 309-346 (2018). ISSN 0021-7824.

12. M. Lighthill and G. Whitham. On kinematic waves. II. A theory of traffic flow on long crowded roads. In Royal Society of London. Series A, Mathematical and Physical Sciences, vol. 229, pp. 317-345 (1955).

13. P. I. Richards, Shock waves on the highway, Operations Research. 4(1), pp. 42-51 (1956). ISSN 0030364X.

14. M. Di Francesco and M. D. Rosini, Rigorous derivation of nonlinear scalar conservation laws from follow-the-leader type models via many particle limit, Arch. Ration. Mech. Anal. 217(3), 831-871 (2015). ISSN 0003-9527.

15. A. Aw and M. Rascle, Resurrection of "second order" models of traffic flow, SIAM Journal on Applied Mathematics. 60(3), 916-938 (2000).

16. H. Zhang, A non-equilibrium traffic model devoid of gaslike behavior, Transportation Research Part B: Methodological. 36(3), 275-290 (2002). ISSN 0191-2615.

17. M. Di Francesco, S. Fagioli, and M. D. Rosini, Many particle approximation of the Aw-Rascle-Zhang second order model for vehicular traffic, Math. Biosci. Eng. 14(1), 127-141 (2017). ISSN 1547-1063.

18. B. Andreianov and M. D. Rosini. Microscopic selection of solutions to scalar conservation laws with discontinuous flux in the context of vehicular traffic. preprint (Dec. 2019).

19. D. C. Gazis, R. Herman, and R. W. Rothery, Nonlinear follow-the-leader models of traffic flow, Operations Research. 9(4), 545-567 (1961). ISSN 0030364X, 15265463.

20. S. Gopal and T. R. Smith. Navigator: An al-based model of human way-finding in an urban environment. In Spatial Choices and Processes, pp. 169-200. Elsevier (1990).

21. D. Helbing and P. Molnár, Social force model for pedestrian dynamics, Phys. Rev. E. 51, 4282-4286 (May, 1995).

22. M. Muramatsu, T. Irie, and T. Nagatani, Jamming transition in pedestrian counter flow, Physica A: Statistical Mechanics and its Applications. 267(3), 487 - 498 (1999). ISSN 0378-4371.

23. J. Dijkstra, H. J. P. Timmermans, and A. J. Jessurun. A multi-agent cellular automata system for visualising simulated pedestrian activity. In eds. S. Bandini and T. Worsch, Theory and Practical Issues on Cellular Automata, pp. 29-36, Springer London, London (2001). ISBN 978-1-4471-0709-5.

24. N. Bellomo and A. Bellouquid. On the modelling of vehicular traffic and crowds by kinetic theory of active particles. In Mathematical modeling of collective behavior in socio-economic and life sciences, Model. Simul. Sci. Eng. Technol., pp. 273-296. Birkhäuser Boston, Boston, MA (2010).

25. P. Degond, C. Appert-Rolland, J. Pettré, and G. Theraulaz, Vision-based macroscopic pedestrian models, Kinet. Relat. Models. 6(4), 809-839 (2013). ISSN 19375093.

26. A. Lachapelle and M.-T. Wolfram, On a mean field game approach modeling congestion and aversion in pedestrian crowds, Transportation Research Part B: Methodological. 45(10), 1572 - 1589 (2011). ISSN 0191-2615.

27. R. L. Hughes, A continuum theory for the flow of pedestrians, Transportation Research Part B: Methodological. 36(6), 507 - 535 (2002). ISSN 0191-2615.

28. N. Bellomo and C. Dogbé, On the modelling crowd dynamics from scaling to hyperbolic macroscopic models, Math. Models Methods Appl. Sci. 18(suppl.), 1317-1345 (2008). ISSN 0218-2025.

29. Y.-Q. Jiang, S.-G. Zhou, and F.-B. Tian, A higher-order macroscopic model for bi-direction pedestrian flow, Phys. A. 425, 69-78 (2015). ISSN 0378-4371.

30. B. Piccoli and A. Tosin, Pedestrian flows in bounded domains with obstacles, Contin. Mech. Thermodyn. 21(2), 85-107 (2009). ISSN 0935-1175.

31. B. Maury, A. Roudneff-Chupin, and F. Santambrogio, A macroscopic crowd motion model of gradient flow type, Math. Models Methods Appl. Sci. 20(10), 1787-1821 (2010). ISSN 0218-2025.

32. C. M. Dafermos, Polygonal approximations of solutions of the initial value problem for a conservation law, J. Math. Anal. Appl. 38, 33-41 (1972). ISSN 0022-247X.

33. A. Bressan, Hyperbolic systems of conservation laws. vol. 20, Oxford Lecture Series in Mathematics and its Applications, Oxford University Press, Oxford (2000). ISBN 
0-19-850700-3.

34. H. Holden and N. H. Risebro, Front tracking for hyperbolic conservation laws, second edn. vol. 152, Applied Mathematical Sciences, Springer, Heidelberg (2015). ISBN 9783-662-47506-5; 978-3-662-47507-2.

35. R. Courant and K. O. Friedrichs, Supersonic flow and shock waves. Springer-Verlag, New York-Heidelberg (1976). Reprinting of the 1948 original, Applied Mathematical Sciences, Vol. 21.

36. P. Baiti and H. K. Jenssen, Blowup in $L^{\infty}$ for a class of genuinely nonlinear hyperbolic systems of conservation laws, Discrete Contin. Dynam. Systems. 7(4), 837-853 (2001). ISSN 1078-0947.

37. S. N. Kruzhkov, First order quasilinear equations with several independent variables, Mat. Sb. (N.S.). 81 (123), 228-255 (1970).

38. G.-Q. Chen and M. Rascle, Initial layers and uniqueness of weak entropy solutions to hyperbolic conservation laws, Arch. Ration. Mech. Anal. 153(3), 205-220 (2000). ISSN 0003-9527.

39. M. Di Francesco, S. Fagioli, and M. D. Rosini, Deterministic particle approximation of scalar conservation laws, Boll. Unione Mat. Ital. 10(3), 487-501 (2017). ISSN $1972-$ 6724.

40. M. Di Francesco, S. Fagioli, M. D. Rosini, and G. Russo. Follow-the-leader approximations of macroscopic models for vehicular and pedestrian flows. In Active particles. Vol. 1. Advances in theory, models, and applications, Model. Simul. Sci. Eng. Technol., pp. 333-378. Birkhäuser/Springer, Cham (2017).

41. M. Di Francesco, S. Fagioli, M. D. Rosini, and G. Russo. A deterministic particle approximation for non-linear conservation laws. In Theory, numerics and applications of hyperbolic problems. I, vol. 236, Springer Proc. Math. Stat., pp. 487-499. Springer, Cham (2018).

42. M. Di Francesco, S. Fagioli, M. D. Rosini, and G. Russo, Deterministic particle approximation of the Hughes model in one space dimension, Kinet. Relat. Models. 10 (1), 215-237 (2017). ISSN 1937-5093.

43. M. D. Rosini. Systems of conservation laws with discontinuous fluxes and applications to traffic. preprint (Nov., 2019).

44. M. Di Francesco and G. Stivaletta, Convergence of the follow-the-leader scheme for scalar conservation laws with space dependent flux, Discrete Contin. Dyn. Syst. 40(1), 233-266 (2020). ISSN 1078-0947.

45. J. Ridder and W. Shen, Traveling waves for nonlocal models of traffic flow, Discrete Contin. Dyn. Syst. 39(7), 4001-4040 (2019). ISSN 1078-0947.

46. M. Di Francesco, S. Fagioli, and E. Radici, Deterministic particle approximation for nonlocal transport equations with nonlinear mobility, J. Differential Equations. 266 (5), 2830-2868 (2019). ISSN 0022-0396.

47. R. Borsche and A. Meurer, Microscopic and macroscopic models for coupled car traffic and pedestrian flow, $J$. Comput. Appl. Math. 348, 356-382 (2019). ISSN 03770427.

48. S. Fagioli and E. Radici, Solutions to aggregationdiffusion equations with nonlinear mobility constructed via a deterministic particle approximation, Math. Models Methods Appl. Sci. 28(9), 1801-1829 (2018). ISSN 02182025.

49. W. Shen, Traveling wave profiles for a follow-the-leader model for traffic flow with rough road condition, Netw. Heterog. Media. 13(3), 449-478 (2018). ISSN 1556-1801.

50. H. Holden and N. H. Risebro, Follow-the-leader models can be viewed as a numerical approximation to the Lighthill-Whitham-Richards model for traffic flow, Netw. Heterog. Media. 13(3), 409-421 (2018). ISSN 1556-1801.

51. N. Forcadel, W. Salazar, and M. Zaydan, Specified homogenization of a discrete traffic model leading to an effective junction condition, Commun. Pure Appl. Anal. 17 (5), 2173-2206 (2018). ISSN 1534-0392.

52. W. Shen and K. Shikh-Khalil, Traveling waves for a microscopic model of traffic flow, Discrete Contin. Dyn. Syst. 38(5), 2571-2589 (2018). ISSN 1078-0947.

53. M. Briani, E. Cristiani, and E. Iacomini, Sensitivity analysis of the LWR model for traffic forecast on large networks using Wasserstein distance, Commun. Math. Sci. 16(1), 123-144 (2018). ISSN 1539-6746.

54. F. Berthelin and P. Goatin, Particle approximation of a constrained model for traffic flow, NoDEA Nonlinear Differential Equations Appl. 24(5), Art. 55, 16 (2017). ISSN 1021-9722.

55. M. Garavello and B. Piccoli, Boundary coupling of microscopic and first order macroscopic traffic models, NoDEA Nonlinear Differential Equations Appl. 24(4), Art. 43, 18 (2017). ISSN 1021-9722.

56. N. Forcadel, W. Salazar, and M. Zaydan, Homogenization of second order discrete model with local perturbation and application to traffic flow, Discrete Contin. Dyn. Syst. 37(3), 1437-1487 (2017). ISSN 1078-0947.

57. P. Goatin and F. Rossi, A traffic flow model with nonsmooth metric interaction: well-posedness and micromacro limit, Commun. Math. Sci. 15(1), 261-287 (2017). ISSN 1539-6746.

58. E. Cristiani and S. Sahu, On the micro-to-macro limit for first-order traffic flow models on networks, Netw. Heterog. Media. 11(3), 395-413 (2016). ISSN 1556-1801.

59. N. Forcadel and W. Salazar, Homogenization of second order discrete model and application to traffic flow, Differential Integral Equations. 28(11-12), 1039-1068 (2015). ISSN 0893-4983.

60. B. Temple, Systems of conservation laws with invariant submanifolds, Trans. Amer. Math. Soc. 280(2), 781-795 (1983). ISSN 0002-9947.

61. B. Andreianov, C. Donadello, and M. D. Rosini, A second-order model for vehicular traffics with local point 
constraints on the flow, Math. Models Methods Appl. Sci. 26(4), 751-802 (2016). ISSN 0218-2025.

62. R. M. Colombo and P. Goatin, A well posed conservation law with a variable unilateral constraint, J. Differential Equations. 234(2), 654-675 (2007). ISSN 0022-0396.

63. T. Liard and B. Piccoli, Well-posedness for scalar conservation laws with moving flux constraints, SIAM J. Appl. Math. 79(2), 641-667 (2019). ISSN 0036-1399.

64. C. Chalons, M. L. Delle Monache, and P. Goatin, A conservative scheme for non-classical solutions to a strongly coupled PDE-ODE problem, Interfaces Free Bound. 19 (4), 553-570 (2017). ISSN 1463-9963.

65. E. Dal Santo, M. D. Rosini, N. Dymski, and M. Benyahia, General phase transition models for vehicular traffic with point constraints on the flow, Math. Methods Appl. Sci. 40(18), 6623-6641 (2017). ISSN 0170-4214.

66. S. Villa, P. Goatin, and C. Chalons, Moving bottlenecks for the Aw-Rascle-Zhang traffic flow model, Discrete Contin. Dyn. Syst. Ser. B. 22(10), 3921-3952 (2017). ISSN 1531-3492.

67. M. L. Delle Monache and P. Goatin, Stability estimates for scalar conservation laws with moving flux constraints, Netw. Heterog. Media. 12(2), 245-258 (2017). ISSN 15561801.

68. B. Andreianov, C. Donadello, U. Razafison, and M. D. Rosini, Riemann problems with non-local point constraints and capacity drop, Math. Biosci. Eng. 12(2), 259-278 (2015). ISSN 1547-1063.

69. C. Chalons, P. Goatin, and N. Seguin, General constrained conservation laws. Application to pedestrian flow modeling, Netw. Heterog. Media. 8(2), 433-463 (2013). ISSN 1556-1801.

70. R. M. Colombo and M. D. Rosini, Existence of nonclassical solutions in a pedestrian flow model, Nonlinear Anal. Real World Appl. 10(5), 2716-2728 (2009). ISSN 14681218.

71. M. D. Rosini, Nonclassical interactions portrait in a macroscopic pedestrian flow model, J. Differential Equations. 246(1), 408-427 (2009). ISSN 0022-0396.

72. P. G. LeFloch, Hyperbolic systems of conservation laws. Lectures in Mathematics ETH Zürich, Birkhäuser Verlag, Basel (2002). ISBN 3-7643-6687-7.

73. M. D. Rosini, Macroscopic models for vehicular flows and crowd dynamics: theory and applications. Understanding Complex Systems, Springer, Heidelberg (2013). ISBN 978-3-319-00154-8; 978-3-319-00155-5.

74. D. Helbing, A. Johansson, and H. Al-Abideen, Dynamics of crowd disasters: An empirical study, Physical Review E (Statistical, Nonlinear, and Soft Matter Physics). 75 (4):046109 (2007). 